\title{
La terciarización en Chile. Desigualdad cultural y estructura ocupacional
}

\author{
Modesto Gayo, María Luisa Méndez y Berta Teitelboim
}

RESUMEN

La terciarización, o transición hacia economías de servicios con un creciente protagonismo de las ocupaciones no manuales, ha sido destacada como un fenómeno central de las sociedades contemporáneas. Frente al supuesto hundimiento numérico y político de los sectores obreros tradicionales, la clase media ha aparecido como la clase social protagónica de las últimas décadas. Vinculada a esta comprensión de la evolución de la sociedad, se ha manifestado un creciente interés por formas de diferenciación social distintas a la ocupacional de las sociedades industriales, entre ellas la cultural. En este trabajo se reconstruye brevemente este debate, y se muestra que si bien pudiera existir un crecimiento de ocupaciones no manuales, y con ello podría pensarse que habría un progresivo avance hacia una sociedad de clase media, hay importantes hallazgos que dificultan simplemente aceptar la afirmación de que Chile se ha convertido en tal tipo de sociedad mesocrática.

PALABRAS CLAVE

CLASIFICACIÓN JEL

AUTORES
Empleo, sector terciario, aspectos culturales, clase media, desarrollo cultural, igualdad, Chile

A14, Y80, Z19

Modesto Gayo es Profesor Asociado en la Escuela de Sociología de la Universidad Diego Portales, Santiago, Chile.modesto.gayo@udp.cl

María Luisa Méndez es Profesora Asociada en la Escuela de Sociología de la Universidad Diego Portales, Santiago, Chile. marialuisa.mendez@udp.cl

Berta Teitelboim es Profesora Asociada en la Escuela de Sociología de la Universidad Diego Portales, Santiago, Chile. berta.teitelboim@udp.cl 


\section{I}

\section{Introducción}

El advenimiento de la sociedad postindustrial, anunciada desde los años sesenta del siglo pasado por insignes intelectuales, como Daniel Bell (1974) y John Galbraith (1967), habría tenido varias consecuencias. La principal consistiría en una transformación del aparato productivo de las sociedades más avanzadas, transitándose, más o menos abruptamente, de una sociedad industrial o de producción de bienes, a una postindustrial o de producción de servicios. Esta transición habría traído consigo otra consecuencia, cual es la reconfiguración de la estructura social. De una sociedad eminentemente obrera, donde primaban el trabajo o las ocupaciones manuales, y en la que debido a ello el socialismo político jugó un papel clave como representante de sus intereses, se pasó a una sociedad en que la pertenencia de clase se volvió difusa ante el abandono, por buena parte de los hijos de la clase obrera, de los patrones de vida que la habían caracterizado. En otras palabras, la sociedad postindustrial produjo el advenimiento de la sociedad de la clase media.

La clase media sería un agregado variopinto de profesionales, gerentes, técnicos y administradores (Gayo, 2013a y 2013c), cuya apariencia poco se parecería a la de los miembros de la clase obrera ${ }^{1}$. No obstante, independientemente de sus diferencias internas (Gayo,

$\square$ La realización de este trabajo fue apoyada económicamente por el Gobierno de Chile a través del proyecto FONDECYT "Capital cultural y territorio en Chile. La estructuración territorial del espacio social más allá de los capitales" ( ${ }^{\circ}$ 1130098).

María Luisa Méndez desarrolló parte de este artículo con el apoyo del Centro CONICYT/FONDAP/15130009.

Asimismo, los autores agradecen el importante apoyo prestado para la realización de este estudio por parte de Francisca Romero y Sara Correa, ambas sociólogas y ayudantes de investigación.

${ }^{1}$ Dentro del marco histórico de las sociedades industriales, las clases sociales han sido habitualmente pensadas y configuradas a partir de la estructura ocupacional. Desde el punto de vista de los esquemas de clase más contemporáneos y de amplio uso en la tradición sociológica de las últimas tres décadas, se encuentra que - de acuerdo con el pensamiento marxista - la clase obrera ha sido caracterizada como un conjunto de ocupaciones no propietarias, de bajo nivel en las organizaciones y con limitada formación intelectual requerida para su desempeño. Mientras que la clase media sería igualmente empleada, pero con mayor formación educativa y niveles organizativos más elevados (Wright, 1983 y fundamentalmente 1994). Por su parte, con un abordaje que comúnmente ha sido entendido como más weberiano, Erikson y Goldthorpe (1993) concebirían a la clase obrera como equivalente a las ocupaciones manuales, de menor prestigio; en tanto que las clases medias serían un conjunto de ocupaciones que podrían ir desde niveles intermedios o de rutina no manual, hasta la que denominan clase de servicio, que incluiría también a sectores altos de la sociedad en los niveles de servicio más elevados.
Teitelboim y Méndez, 2013), el énfasis en la importancia de esta nueva clase tuvo, como efecto, el progresivo abandono del interés por la clase obrera. Esta creciente marginalización de dicha clase, que ahora cumplía el mismo papel que Marx le había atribuido a la clase media propietaria en los inicios del capitalismo (Marx y Engels, 1971), es decir, vista en un comienzo como protagonista de la historia, para pasar a ser una categoría social residual o propia del régimen capitalista anterior, no solo estaría vinculada al éxito - tanto social como discursivo - de la clase media, sino a un proceso más profundo: la aparente disolución de la estructura de clases que había caracterizado a la sociedad industrial desde su mismo origen (Pakulski y Waters, 1996; Beck y Beck-Gernsheim, 2002).

En relación con este amplio marco de análisis, durante las últimas dos décadas ha habido una sostenida revitalización de los estudios de estratificación social en América Latina, y una parte importante de esas investigaciones se ha enfocado en la definición, delimitación y medición de las clases medias (Filgueira, 2001; Atria, Franco y León, 2007; entre otros). En este conjunto de estudios se ha expuesto el crecimiento de los sectores medios en países como el Brasil (O’Dougherty, 2002), Chile (León y Martínez, 2004) y México (Torche y López-Calva, 2013), entre otros, apuntando principalmente al cambio en la estructura social derivado del llamado proceso de terciarización o transición a una economía de servicios. De este modo, los argumentos han girado en torno del engrosamiento del sector no manual (Filgueira, 2001; Atria, 2004), la expansión en la capacidad de consumo (Knowledge Wharton, 2008), y el aumento en los niveles de ingreso (Ferreira y otros, 2013) y de los niveles educativos (Daude, 2012). Asimismo, en algunos estudios se ha vinculado el crecimiento de los estratos medios con el fortalecimiento de los sistemas democráticos (Paramio y Güemes, 2013)², así como con los procesos de desarrollo económico (Ferreira y otros, 2013). Sin embargo, no existe consenso acerca de si efectivamente estamos en presencia de una transformación

\footnotetext{
2 Paramio y Güemes (2013) señalan que los países con una clase media amplia y fortalecida tienden a tener democracias más sólidas, mejor gobernanza, un sistema crediticio estable y un mayor gasto en salud pública y educación. En este sentido, la clase media jugaría un papel fundamental en el desarrollo económico y la estabilidad política.
} 
general de la región latinoamericana hacia sociedades de clase media. Por el contrario, existe un cuerpo de literatura que se muestra renuente a aceptar radicalmente este fenómeno: sin perjuicio de que las clases medias gocen de mejoras respecto de sí mismas y de los grupos más pobres, aún serían estratos vulnerables que no han logrado consolidar su posición estructural a través del tiempo (OCDE, 2011; López-Calva y Ortiz-Juárez, 2012; Paramio y Güemes, 2013).

Una dimensión adicional en el campo de estudios de las clases medias a nivel global es la que se refiere al ámbito cultural, entendido este como patrones de gusto y participación cultural, que en la investigación sociológica de las últimas tres décadas se ha abordado desde una matriz fundamentalmente bourdieuana (Bourdieu, 1979). En estos estudios, la clase media no solo se define a partir de sus ocupaciones, niveles educativos o de ingresos, sino que es caracterizada como un grupo social culturalmente activo o con una notable propensión a tener una actividad cultural intensa (Bennett y otros, 2009; Bennett, Bustamente y Frow, 2013; Gerhards, Hans y Mutz, 2013; Roberts, 2004). Junto con este activismo relativo a las prácticas de alta cultura, algunos autores sostienen que en dicho activismo se incluirían prácticas culturales más masivas, lo que podría implicar un debilitamiento de las fronteras simbólicas de clase, motivado por la creciente adopción por parte de sus miembros de prácticas culturales habitualmente consideradas más próximas a los hábitos y gustos de sectores más populares (Peterson y Kern, 1996).

Desde un punto de vista explicativo, en estas investigaciones se han utilizado comúnmente esquemas o clasificaciones ocupacionales de clase que han mostrado reiteradamente su relevancia para entender las desigualdades culturales. Al respecto, en lugar de optar por una clasificación de clase particular (Gerhards, Hans y Mutz, 2013), donde de antemano se proponen agrupaciones ocupacionales que tienen que ver con otros estudios, en este trabajo se adopta una perspectiva bourdieuana, según la cual las agrupaciones ocupacionales son un producto inductivo como resultado del estudio anterior de las similitudes entre individuos derivadas del comportamiento cultural. En otros términos, aquí la contribución a una evaluación del estado actual de la sociedad chilena pasa necesariamente por el estudio de los patrones culturales, y la ocupación vendría posteriormente como una expresión de los complejos anclajes estructurales a los que aquellos están sujetos (Pinto, 2013). Más que imponerle a la participación cultural ciertos determinantes, como puede ser el de clase (ocupacional), un elemento constitutivo de esta aproximación consiste en abrir la posibilidad de que eventualmente haya otras líneas divisorias tanto o más relevantes, que quizás pudieran servir para dejar en un segundo plano la interpretación clasista de la sociedad.

Este trabajo se hace eco de estos debates, agregando, como ya se ha mencionado, una dimensión clave en la comprensión de la formación de las clases medias en América Latina: las prácticas de participación y gusto culturales. La hipótesis del presente estudio es que si la terciarización se ha instalado en Chile, y este país se ha convertido en una sociedad en que predomina la clase media, ello debería verse acompañado de patrones de participación cultural que expresen tal realidad. De forma destacada, aquí se atiende principalmente a los siguientes. En primer lugar, se observa la naturaleza de los perfiles de participación cultural y la importancia porcentual aproximada de dichos tipos, de tal manera de evaluar los grados y el peso relativo del activismo cultural. En segundo lugar, se estudia la relación entre tal activismo y un conjunto de variables que se esperaría influyeran en él, tales como el nivel educativo, el grupo socioeconómico, la ocupación, la edad, el territorio y el sexo. A este respecto, en una sociedad de clase media sería de esperar una significativa erosión de las fronteras culturales o simbólicas de clase, es decir, se esperaría encontrar variables tan importantes como la que distingue según el nivel socioeconómico y, en esta misma línea, también según la ocupación. Sin embargo, una vez analizados los datos, los resultados sobre la participación cultural en Chile muestran una sociedad claramente diferenciada y con rasgos de polarización. Sintéticamente, por una parte se encuentra una mayoría compuesta por personas con ocupaciones manuales y no manuales de bajas remuneraciones y restringidos niveles educativos, y por otra, un grupo cuyas ocupaciones reciben mayores remuneraciones y suelen exigir niveles educativos más elevados. En este sentido, el modelo de postindustrialización europea o de terciarización descrito para el caso latinoamericano debe ser explorado de manera compleja y no darse por sentado como una cuestión de inercia histórica. El Chile actual es un país marcadamente desigual, y su desigualdad se hace evidente a través de las prácticas culturales de sus ciudadanos, lo que denota más bien la imagen de un país con serias limitaciones desde el punto de vista de su desenvolvimiento cultural y, por extensión, social.

A objeto de desarrollar estos argumentos, en este artículo se utilizan los datos de la Segunda Encuesta Nacional de Participación y Consumo Cultural realizada por el Consejo Nacional de la Cultura y las Artes (CNCA) en el año 2009. Este estudio se llevó a cabo en las 15 
regiones de Chile a personas residentes mayores de 15 años, encuestándose finalmente a 4.000 personas dentro de esta población. En el cuestionario de la encuesta se abordaron diferentes temáticas, entre las que están: la asistencia a espectáculos culturales, la lectura de libros y actividades afines, la tenencia en el hogar de equipamiento cultural, la utilización de los medios de comunicación, la participación en actividades relativas al patrimonio cultural y la realización de actividades culturales y artísticas (CNCA, 2011a).
Luego de la presente Introducción, el documento se organiza de acuerdo con la siguiente estructura: en la sección II se aborda el desarrollo de la investigación en Chile. A continuación, la tercera sección se refiere a las actividades estudiadas y los métodos de investigación. A su vez, en la sección IV se investiga la desigualdad cultural en la sociedad chilena, en tanto que la quinta sección está destinada al estudio de la cultura, la ocupación y la terciarización. Finalmente, en la sección VI se entregan las conclusiones del trabajo.

\section{II}

\section{La investigación en Chile}

En Chile ha venido exacerbándose en los últimos años el interés, tanto mediático como académico, en entender la sociedad como una en que predomina la clase media. Sin embargo, las bases sobre las que ha tomado forma este planteamiento son diferentes a las que permitieron el surgimiento de estas ideas en los casos de los países más desarrollados. Por una parte, en el caso chileno venía poniéndose énfasis en el tema de la pobreza. Una vez que se constató que el país había progresado social y económicamente, y que los índices de pobreza habían experimentado una importante reducción cuantitativa, se produjo un giro - desde finales de los años noventahacia la interpretación de la sociedad desde la presencia de la clase media, lo que venía siendo habitual en los países más avanzados desde algunas décadas atrás. Esto es importante porque supuso, en un viaje intelectual acelerado, transitar no entre la industrialización obrera y la postindustrialización de clase media, sino entre la pobreza y la clase media, sin solución de continuidad alguna $^{3}$. Por otra parte, no solo la desigualdad en Chile continúa constituyendo un factor cotidiano de estructuración de las relaciones sociales, sino que existe abundante evidencia de que la clase social sigue siendo una variable configuradora clave de los comportamientos de sus ciudadanos ${ }^{4}$.

\footnotetext{
${ }^{3}$ Un intento de reflexión sobre la estratificación en la sociedad chilena actual, posterior al período en que se enfatizó la relevancia de la pobreza, es el artículo de Manuel Canales (2007).

${ }^{4}$ Un ejemplo del reconocimiento tanto de la desigualdad como del enriquecimiento generalizado de la población en Chile, es la aportación de Rasse, Salcedo y Pardo (2009), que plantean la necesidad de repensar la desigualdad y las clasificaciones con las que se la representa ante el evidente cambio que ha experimentado la sociedad chilena en los últimos 20 años.
}

Sea como fuere, no ha crecido únicamente el interés en la clase media (Méndez, 2007 y 2010; Espinoza y Barozet, 2009), sino que también ha venido aumentando la atención prestada al fenómeno de las prácticas culturales (Gayo, Teitelboim y Méndez, 2013). De este modo, se considera que la calidad de la pertenencia a una sociedad debe entenderse no solo desde el éxito económico, sino también a partir de la participación en actividades culturales (CNCA, 2011b; Güell, Peters y Morales, 2012), lo que en ocasiones se ha vinculado al desarrollo humano (PNUD, 2002; Délano, 2011). ¿Cómo ha sido descrito este fenómeno en la literatura orientada al estudio del caso chileno?

Como elemento de información preliminar, debe subrayarse que en Chile se han realizado algunas investigaciones sobre consumo cultural, pero difícilmente puede decirse que, hasta el momento, se haya desarrollado un extenso y profundo debate sobre el tema. Hay algunos trabajos, la mayoría de ellos recientes, cuyos datos proceden principalmente de las encuestas realizadas por el CNCA, quedando todavía un largo camino por recorrer para la formación de un conocimiento bien asentado, profundo y sistemático.

Asimismo, es importante entender dos fenómenos relativos a los estudios recientes sobre las prácticas culturales. Por una parte, hay una notable aceptación del marco de la terciarización para la comprensión de la sociedad chilena actual (Brunner, Barrios y Catalán, 1989). El rechazo del enfoque de clase como aproximación explicativa, la aceptación del progresivo avance hacia una sociedad de clase media, la insistencia en el estatus frente a la clase social, y el reconocimiento de la individualización como un fenómeno clave para 
comprender la sociedad, son evidencia de ello. Por otra parte, si bien en conexión con el punto anterior, ha habido un evidente interés por interpretar la realidad chilena desde agendas de investigación que actualmente están en boga en instituciones de los Estados Unidos de América y de la Europa más desarrollada (Torche, 2007; Nazif, 2007). Con relación a estos aspectos, a continuación se presenta el conocimiento que hasta ahora se ha ido generando, para cuyo propósito se recurre a las principales aportaciones sociológicas al estudio de la práctica cultural en la sociedad chilena de las últimas décadas. Durante este breve recorrido, emergerán algunos de los patrones más relevantes identificados hasta la fecha.

En primer lugar, existe un acuerdo generalizado sobre un fenómeno clave que habría reconfigurado, en las últimas dos o tres décadas, la participación cultural de los chilenos. Se trata de la expansión o masificación del consumo cultural, y no cultural, por lo menos desde comienzos de los años ochenta (Brunner, Barrios y Catalán, 1989; PNUD, 2002; Catalán, 20095). En este sentido, tal y como se recoge en un estudio del Ministerio de Desarrollo Social, el gasto de los hogares chilenos habría crecido significativamente en la década comprendida entre los años 1988 y 1997 (Bernasconi y Puentes, 2001). No obstante, es importante entender que el fenómeno de la masificación ha sido asimétrico, como en su momento observaron Catalán y Sunkel (1990). Para estos autores, habría que distinguir entre dos tipos de actividades masivas. Por una parte, estarían las más masificadas, cuyas prácticas serían propias de todos los grupos y estratos sociales (la televisión, la música, la radio). Por otra, encontraríamos otro tipo de masificación referida a actividades muy comunes, pero cuya práctica tendría una intensidad asociada con los niveles educativos y de ingresos. Entre estas últimas, estarían la lectura de prensa, revistas y libros, y la asistencia al cine.

En segundo lugar, más allá del consenso sobre la creciente masificación de las prácticas culturales, existe también un amplio acuerdo en torno de la constatación de importantes diferencias en el gusto y la participación culturales, de acuerdo con variables sociológicas habitualmente utilizadas en los análisis de este tipo (Catalán y Sunkel, 1990; CNCA, 2007; Torche, 2007; Nazif, 2007; Gayo, Teitelboim y Méndez, 2009 y 2013; Güell y Peters, 2012; Gayo, 2013b). Entre ellas estarían el sexo ${ }^{6}$, la ocupación o el grupo socioeconómico, la

\footnotetext{
5 En este artículo se trata el consumo en general, y no solo el que se refiere a bienes o servicios culturales.

6 En la actualidad, es más común hablar de "género", pero la información que normalmente se recoge se refiere al sexo del encuestado.
}

región de residencia, la edad, la educación y el estatus. Existe, por lo tanto, plena conciencia sobre la desigualdad, o las desigualdades, en el ámbito del consumo o la participación cultural ${ }^{7}$.

A nivel comparado, como un tercer elemento de análisis se ha subrayado que en Chile se observan niveles de participación cultural inferiores a los de muchos otros países de América Latina. En la introducción de un informe reciente del CNCA, se afirma que: "En Chile el consumo cultural es bajo... en especial si nos remitimos a una comparación con países como Argentina, Uruguay, Colombia, México, Brasil" (CNCA, 2007, pág. 6). De alguna manera, si en los principales países de la región se registran mayores niveles de participación, se estaría implicando que el caso chileno es problemático. En otros términos, se entiende que hay una tarea por emprender para alcanzar niveles similares a los de otros países de la región. No obstante, ello se podría afirmar pese a que no es claro que haya evidencia contundente que muestre tal desigualdad en desmedro de Chile (Nivón y Sánchez, 2012; OEI, 2014). Asimismo, en el informe del Ministerio de Desarrollo Social (MIDEPLAN) (Bernasconi y Puentes, 2001) antes aludido se observan dos patrones adicionales en relación con la dimensión comparada. Por una parte, desde el punto de vista de la proporción de gasto en cultura de los hogares, Santiago y Montevideo mostrarían niveles similares, en comparación con un mayor nivel de gasto presente en Buenos Aires. Por otra, esta última ciudad tendría el grado más elevado de desigualdad en el gasto, mientras que Montevideo sería el caso donde el consumo sería más igualitario, estando Santiago entre ambas ciudades en cuanto a gasto en cultura.

En cuarto lugar, y como se ha mostrado anteriormente, ha habido bastante investigación empírica en comparación con un trabajo de desarrollo teórico mucho más débil. La aportación de Brunner, Barrios y Catalán (1989), ya algo antigua a estas alturas y con escaso tratamiento de los datos sobre consumo cultural, es quizás una excepción —al menos por su ambición explicativa- en el marco de una teoría de la modernización con tintes bourdieuanos. A su vez, algunos trabajos de mayor profundidad empírica (Bernasconi y Puentes, 2001; Nazif, 2007; Torche, 2007; Güell y Peters, 2012) son más débiles en el tratamiento de la dimensión teórico-explicativa. Entre estos dos polos, desde el punto de vista de la combinación entre

\footnotetext{
${ }^{7}$ Como limitación a estos estudios de corte cuantitativo, hay aportaciones que señalan que se requiere una aproximación de tipo cualitativo o más etnográfica para contribuir al entendimiento de los procesos de distinción o diferenciación simbólica asociados a la práctica cultural (Aguilar, 2009; Gayo, Teitelboim y Méndez, 2009; Gayo y otros, 2011).
} 
hablar de la sociedad en su conjunto y la profundidad en el análisis de datos, estaría el Informe del Programa de las Naciones Unidas para el Desarrollo (PNUD), donde se incorporan de manera importante metodologías cualitativas, lo que le permite construir un relato más allá de las encuestas (PNUD, 2002).

\section{III}

\section{Actividades estudiadas y métodos de investigación}

Hasta ahora, los estudios en Chile se han caracterizado por al menos tres rasgos. El primero sería que la forma de categorizar las variables ha sido estándar, esto es, se han seguido los patrones habitualmente empleados para este y otros países. Un claro ejemplo de ello es la manera de categorizar a los encuestados sobre la base de clasificaciones de clase bien conocidas, como la de Erikson y Goldthorpe (Torche, 2007; Nazif, 2007). Por lo general, este proceder se ha justificado en virtud de las necesidades de la comparación, sea esta desarrollada o no, y sin prestar demasiada atención a que el patrón a partir del cual se compara suele corresponder al de estudios realizados en un grupo muy pequeño de países altamente desarrollados. El segundo rasgo ha tenido que ver con el uso de técnicas estadísticas, dando prioridad dentro de estas técnicas a aquellas que distinguen entre variables independientes y dependientes, destacándose las regresiones tanto lineales como logísticas. El tercer rasgo, relacionado con el anterior, se refiere a que el tratamiento de los diferentes gustos y prácticas de consumo o participación culturales se ha realizado por separado, lo que significa que no ha sido habitual tener una imagen global de esta dimensión del campo cultural, como consecuencia de la incorporación de un amplio conjunto de variables ${ }^{8}$. Un ejemplo palmario que ilustra este punto es el artículo de F. Torche (2007) sobre la lectura; lo mismo se podría decir sobre los capítulos de los libros editados por Catalán y P. Torche (2005) y Güell y Peters (2012), ambos acerca del tema del consumo cultural.

Con un tratamiento diferente de los datos, en el presente estudio se incorpora un listado muy extenso de actividades y preferencias culturales de acuerdo con la disponibilidad de información contenida en la Encuesta Nacional de Consumo Cultural en Chile,

8 Excepciones a ello han sido los artículos de Gayo, Teitelboim y Méndez (2009 y 2013) y Gayo (2013b). realizada en el año 2009 (véase el cuadro A.1 del anexo 1). Para integrar estas variables en un análisis conjunto, se utiliza una técnica estadística conocida como análisis de correspondencias múltiples, que la obra de Bourdieu — sobre todo a través de su libro más conocido, La distinction (1979)— contribuyó decisivamente a popularizar, especialmente en el ámbito de la sociología. Esta técnica permite representar, en un espacio de $n$ dimensiones (normalmente se elegirán las dos más relevantes estadísticamente), las posiciones de los encuestados definidas a partir de sus gustos y comportamientos culturales. Ello posibilita conocer las distancias relativas entre estos últimos. Asimismo, se puede entender que un conjunto de preferencias y actividades similares, relativamente próximas en el espacio, representará un estilo de vida diferenciado. Además del estudio de la cercanía entre prácticas y gustos, el análisis de correspondencias múltiples también permite explorar cuáles pueden ser las variables que estructuran o influyen en la configuración de ese espacio social particular, o - en otros términos- qué variables pudieran contribuir a explicar los diferentes estilos de vida o patrones de participación cultural. Tanto para esta tarea como para la anterior, en principio, no hay más límite que el de disponer de variables categóricas 9 .

Como en este estudio existe un interés particular en la terciarización, la reflexión sobre la variable ocupación tiene un papel particularmente importante. En este sentido, pareció conveniente mantener la variable ocupación lo más desagregada posible. De esta manera, se podría replantear una discusión sobre la estructura social del Chile actual, a partir de un trabajo de investigación con un marcado componente inductivo. La idea era, en definitiva, avanzar hacia una evaluación del entendimiento

\footnotetext{
${ }^{9}$ Dos ejemplos de uso de esta técnica estadística son Gayo, Savage y Warde (2006), para el caso británico, y Gayo, Teitelboim y Méndez (2009), para Chile.
} 
de Chile como una sociedad terciarizada, pero acotada al área del gusto y la participación culturales.

Cuando se habla de ocupación, es importante subrayar que aquí no se hace referencia a proporcionar o construir un esquema de clase, como los que usualmente se han venido empleando en sociología por parte de los especialistas que se han dedicado al estudio de la estructura social. Se debe dejar en claro que esto será así solo de manera parcial. ¿Por qué parcial? Porque para clasificar a los encuestados, en este trabajo se parte — como ha sido común- de la Clasificación Internacional Uniforme de Ocupaciones (CIUO-88) de la Organización Internacional del Trabajo (OIT), pero con la diferencia de que aquí se intenta dejar la clasificación tan desagregada como sea posible (véase el cuadro A.1 del anexo 2). Esto dejó fuera, en un primer momento, a todos los que no tenían una ocupación registrada o que carecían de ella, generalmente por formar parte de la población que con frecuencia ha sido denominada "pasiva" (jubilados, estudiantes, dueñas de casa y otros). Como respuesta a este hecho, y también para evitar utilizar clases mediadas, esto es, asignadas de acuerdo con la del jefe de hogar, se produjo una clasificación de los que carecían de ocupación, es decir, de aquellas personas no clasificadas, o clasificadas recurrentemente desde su "pasividad". Con miras a su clasificación, se distinguió a los desocupados, los estudiantes, los jubilados y las dueñas de casa, y se les asignó una categoría según la combinación entre su nivel de estudios y los ingresos del hogar, ambas variables con cuatro categorías. Como resultado de este cruce, las alternativas de clasificación con menos de 20 casos fueron reunidas bajo la categoría "otro" y excluidas del análisis. Esto significa que la ocupación sirvió como un criterio clasificador en un principio, pero tuvo que ser complementado con otros criterios para que todos los individuos encuestados tuvieran su propia categoría de clase (véase el cuadro A.1 del anexo 2), es decir, al menos una que fuera inteligible y sociológicamente sustantiva desde el punto de vista del entendimiento de las desigualdades que también manifiestan las ocupaciones (Pinto, 2013).

Con ello se trata de dar respuesta a los siguientes problemas. En primer lugar, el hecho de que en Chile el llamado trabajo "informal" es más masivo o generalizado que en los países con mayor desarrollo industrial. En segundo lugar, se quiere tener la capacidad de observar lo que ocurre en el interior de las clases obrera y media, sin partir de su homogeneidad interna en el caso de la primera, o de una particular heterogeneidad, basada especialmente en la distinción entre directivos y profesionales, respecto de la segunda. Finalmente, también parece importante, dado sobre todo el cambio que ha habido en las familias (tasas de divorcio, relación con y cuidado de los adultos mayores, entre otros), incluir a las personas no ocupadas con su propia posición de clase, lo que se hizo - como fue mencionado- sobre la base de la educación personal y el ingreso del hogar.

\section{IV}

\section{Desigualdad cultural}

En Chile existen patrones de participación cultural bien definidos, que tienen un estrecho vínculo con variables estructurales, tales como la ocupación, los ingresos, la educación y la edad. A este respecto, es imprescindible entender que dichos patrones son sobre todo el resultado de la combinación de los capitales económico y educativo, ambos estrecha y positivamente correlacionados, junto con la edad.

¿Cuántos y cuáles son esos patrones de participación y gustos culturales, y a qué variables sociodemográficas están asociados? A partir de un análisis de conglomerados, que aquí no se presenta por razones de espacio, se han identificado seis tipos de perfiles culturales, que fueron obtenidos mediante el uso de los ejes de coordenadas derivados de la realización de un análisis de correspondencias múltiples. El mapa original de prácticas al que se refieren dichas coordenadas puede verse en el gráfico A.1 del anexo 2, donde es posible estudiar la asociación relativa entre las categorías, es decir, que cuanto más cerca se encuentran es más probable que un individuo realice ambas prácticas, siempre en términos relativos a terceras alternativas. Asimismo, en el gráfico A.2 del anexo 2 se exploran las variables que pudieran estar asociadas a los ejes del mapa o espacio de categorías anterior, destacándose las variables antes mencionadas: niveles educativo y socioeconómico, por una parte, y edad, por otra. El gráfico A.3 del anexo 2 permite visualizar los lugares promedio en el espacio social y el tamaño relativo de los seis perfiles culturales antes aludidos, construidos sobre la base de las coordenadas 
del gráfico A.1 del anexo 2. Mediante el análisis de los patrones de activismo cultural que se presentan a continuación, se trata de sintetizar la información de los gráficos A.1, A.2 y A.3 del anexo 2, puesto que es el mismo espacio social y geométrico.

El primer patrón concentraría un 6,8\% de la muestra y estaría representado por un individuo que visita sitios patrimoniales, asiste a conferencias, viaja fuera del país, va a museos y al teatro con cierta frecuencia, asiste el cine y posee una importante cantidad de libros. Se trata preferentemente de un profesional o empleado de oficina joven, con estudios universitarios o de nivel superior técnico, y perteneciente a un grupo socioeconómico $\mathrm{ABC} 1$ o $\mathrm{C} 2$.

El segundo patrón, donde se situaría un 9,6\% de las personas, estaría representado por una persona que se destaca por su acumulación de objetos culturales, entre los cuales estarían los libros de colección, las pinturas originales, los grabados y las esculturas. Presenta rasgos sociodemográficos similares a los del primer patrón, pero quizás se está ante un profesional con algo más de trayectoria laboral y, por lo tanto, más asentado económicamente. Estos dos patrones de comportamiento son los que se podrían catalogar de "elitistas" o de "élite cultural".

El tercer patrón, cualitativamente diferente de los dos anteriores, tendría un perfil de activismo masivo. En él destacan actividades como los espectáculos de malabarismo, pantomima y magia, la asistencia a parques de diversiones, los viajes dentro del país, las visitas al zoológico, la comunicación por Internet y la asistencia al estadio, entre otras. Es un patrón compartido por un $8,5 \%$ de los encuestados, y se trataría mayormente de jóvenes con formación superior técnica o media, de grupos socioeconómicos $\mathrm{C} 3$ o superiores.

Por su parte, el cuarto patrón sería la versión popular del patrón previo, es decir, mostraría más orientación a la no participación en las actividades propuestas y, de igual modo, similitudes en las preferencias. Entre estas últimas se encuentran: la frecuencia de ver películas de video o DVD, la asistencia al estadio, la tenencia de un número razonable de libros y el gusto por la música rock. Este patrón concentraría a un $27,1 \%$ de la muestra. También se trataría de un conjunto preferentemente joven, aunque algo más adulto que el del patrón anterior, de estrato un poco más bajo, aunque también tendría como extremo inferior al grupo socioeconómico C3. En este destacarían actividades u ocupaciones como los conductores, los trabajadores de los servicios personales, los técnicos y operadores, y las dueñas de casa con estudios medios y de hogares con ingresos de al menos entre $\$ 250.000$ y
$\$ 500.000$ mensuales. Además, se trataría de un patrón más bien masculino.

Finalmente, el quinto y sexto patrones corresponden a los que representan más genuinamente el mundo popular, o los que en otro estudio eran catalogados como los representantes de la inactividad y la pasividad cultural (Gayo, Teitelboim y Méndez, 2009), desde el punto de vista de la participación en las actividades relacionadas con la cultura que han resultado de interés para las encuestas de consumo cultural en Chile. En lo que respecta al quinto patrón, en el que se concentra el 28,5\% de los casos, es decir, el grupo más grande de todos, se destaca su pasividad. En efecto, sus representantes poseen pocos libros, prefieren aquellos de contenido religioso, y el tipo de cine que admiran es el de las películas románticas. Desde el punto de vista sociodemográfico, son personas de mediana edad o adultos mayores — mujeres en su mayoría — con escasa educación y niveles socioeconómicos D o E, donde resaltan el trabajo no calificado, las dueñas de casa y los jubilados con formación media o básica, cuyos ingresos del hogar, en ambos casos, son inferiores a $\$ 250.000$ mensuales. Por su parte, el sexto patrón comprende a un $19,6 \%$ de los encuestados y tiene un claro perfil de inactividad. Si por algo sobresale, igual que el grupo anterior aunque todavía más notoriamente, es por su muy escaso grado de involucramiento en el ámbito del consumo o la participación cultural. Sus rasgos sociodemográficos y ocupacionales presentan el perfil esperado, esto es: bajo nivel educativo y económico, elevado grado de envejecimiento de su población y trabajo no calificado. Además, en términos generales, cabe prestar atención al hecho de que los perfiles que muestran un mayor nivel de activismo cultural suelen estar asociados a la Región Metropolitana, mientras que lo contrario sucede con la inactividad o pasividad cultural ${ }^{10}$.

\footnotetext{
${ }^{10}$ Con los términos "inactividad" y "pasividad" no se quiere significar de ningún modo que los patrones de comportamiento de las personas calificadas con tales adjetivos casi carezcan de actividad cultural en un sentido extenso. Son calificaciones que tienen como punto de referencia principal o más próximo los indicadores provenientes de la encuesta misma. Puede ser un motivo de discusión si las preguntas son demasiado restringidas con respecto a los comportamientos con los que finalmente las personas son clasificadas. En tal sentido, en este trabajo se consideran valiosos los aportes que han hecho las mediciones de encuesta tanto a nivel nacional como internacional, sobre todo durante las últimas cuatro décadas de estudios sobre el comportamiento cultural, tradición con la que entronca esta investigación. Finalmente, el uso de términos como "pasivo" o "inactivo" no pretende responsabilizar a las personas descritas de ese modo. Más bien por el contrario, quiere ser un instrumento que permita entablar un diálogo crítico con una realidad social que muy probablemente las oprime o las deja atrás.
} 
¿En síntesis, qué se puede concluir de la descripción señalada? Básicamente, que el activismo cultural es un comportamiento restringido y múltiple. Por una parte, restringido porque afecta en el mejor de los casos al $50 \%$ de la población. Si se excluye al cuarto patrón, que presenta un activismo muy débil, se hablaría de un $25 \%$, como máximo, dentro del cual el patrón más elitista, que acumularía los porcentajes de los patrones 1 y 2 , estaría en torno del $17 \%$. En otros términos, la mayoría de la población chilena no participa, o lo hace escasamente, en actividades culturales, y ello no solo se refiere a aquellas más elitistas sino, también, a muchas de los frecuentemente considerados eventos masivos. En segundo término se está ante un patrón múltiple, porque hay diferentes perfiles participativos. El involucramiento cultural presenta formas variadas, cuya diversidad tiene que ver, sobre todo, con la acumulación de recursos económicos y educativos. Se podría decir que participar o no es una función de los capitales económico y educativo, de modo que la pauta indicaría que a mayor acumulación de dichos capitales se incrementaría el grado de participación y distinción culturales. A este respecto, es importante tener presente que ambos capitales se correlacionan fuerte y positivamente, lo que dificulta distinguir entre ellos. Por lo tanto, los recursos económicos y culturales explicarían en gran medida la propensión a tomar parte en actividades culturales y a hacerlo en particular en algunas de ellas, o a mostrar cierto tipo de preferencias estéticas o de contenido. Sin embargo, una explicación que se remita únicamente a estos capitales será, necesariamente, incompleta. En tal sentido, los patrones de consumo cultural en Chile no podrían entenderse convenientemente sin la inclusión de la variable etaria ${ }^{11}$ (Gayo, Teitelboim y Méndez, 2009). El tipo de pauta de consumo y gusto se asocia directamente con la edad de las personas. En el cuadro A.2 del anexo 2 se presenta en términos dicotómicos, y con el propósito de simplificar la abundante información aportada anteriormente, la estructura de los patrones de práctica cultural representada por tres principales.

11 No cabe duda de que esto no solo sucede en Chile. Dos estudios en los que también se muestra la relevancia de la edad en el caso del Reino Unido de Gran Bretaña e Irlanda del Norte son los de Bennett y otros (2009) y Gayo (2006).

\section{V}

\section{La cultura, la ocupación y la terciarización}

La sociedad postindustrial o terciarizada nos advertía de un cambio radical que se estaba produciendo en las sociedades avanzadas o más prósperas. El viejo obrero de vestimenta adecuada a su trabajo manual se estaba transformando en un empleado de camisa inmaculada y labor intelectual. Las empresas dejaban de estar tan centradas, como otrora, en la producción de objetos, y se advertía la expansión incontenible de los negocios orientados a los servicios. El principal ingrediente de la nueva industria no eran los recursos naturales para ser transformados, y las fuentes de energía para hacer posible tal transformación. Ahora la materia prima era el intelecto que portaban los recursos humanos.

Todo ello estaba relacionado con dos fenómenos igualmente significativos. Por una parte, parecían estar dándose las condiciones para el triunfo indiscutido de las habilidades cognitivas y de la inteligencia de los individuos. Por fin, se podría dar satisfacción, aunque fuese parcialmente, a una de las grandes promesas de la modernidad, esto es, el logro individual, el éxito personal a través del mérito, el que sería medido y premiado - comúnmente- de acuerdo con el grado de inteligencia práctica de la persona, o el potencial productivo de sus conocimientos. En coherencia con ello, por otra parte, crecería con fuerza un grupo de personas que desde antes había sido conocido como "clase media", y cuya expansión hacía posible pensar en la progresiva conformación de una sociedad donde las diferencias no fuesen producto directo de la estructura industrial-ocupacional.

De esta manera, el marco de la terciarización laboral apuntaba hacia los individuos y no a las clases sociales; hacia la inteligencia y el mérito, y no a la propiedad de los medios de producción; hacia la movilidad social, y no a la reproducción de acuerdo con el origen familiar; hacia la indiferenciación ocupacional, y no a las estructuras jerárquicas evidentes de las sociedades derivadas de los primeros estadios de la industrialización. En otros términos, la sociedad terciarizada podría ser entendida como una etapa más 
avanzada de la modernidad, donde su ideal normativo parecía tener más visos de realización.

¿Es en esta perspectiva, anunciada 40 años atrás, desde donde se puede entender de mejor manera el comportamiento de la sociedad chilena actual, a comienzos del siglo Xxi? Quizás, desde un punto de vista económico, la respuesta sea más ambigua, pero desde el ámbito de la práctica cultural no es ese el modelo con el que nos encontramos. Está lejos de serlo. La terciarización en Chile, de haberse producido, ha ido acompañada de una marcada desigualdad cultural, en parte ya analizada en la sección anterior. A este respecto, esta sección se centra en la dimensión ocupacional o del trabajo, y se presta atención preferente a los resultados que se observan en el gráfico A.4 del anexo 2.

Desde el punto de vista de la estructura ocupacional, la sociedad chilena no parece haberse conformado de acuerdo con un patrón de integración en torno de una gran clase media. Ello no significa negar que se haya producido un enriquecimiento creciente de un, cada vez mayor, número de sus miembros, sino reconocer que predomina un patrón de polarización entre dos grandes grupos de ocupaciones, lo que explicaría —al menos parcialmente- la divisoria entre la participación y la inactividad característica de la estructura del espacio social en este país (Gayo, Teitelboim y Méndez, 2013). En otros términos, existe un grupo de ocupaciones donde estarían los profesionales, de forma destacada, los altos ejecutivos, los técnicos en ingeniería y los empleados de oficina, que conformarían un conjunto que estaría relacionado con la participación cultural en sus versiones más o menos elitistas. Por otra parte, se observa que ocupaciones como los peones del sector primario, los trabajadores no calificados, los conductores, los operarios de las industrias del metal y textil, los operarios de maquinaria, los empleados de comercio y los trabajadores de los servicios personales, compondrían un grupo cuya característica principal sería su baja participación cultural. Dos grandes polos ocupacionales que, sin duda, no sería difícil relacionar con rentas y niveles educativos diferentes, concentrando el segundo grupo medios más bajos que los de las ocupaciones mencionadas en primer lugar. Por lo tanto, se puede entender que en Chile existen dos grandes conjuntos o polos ocupacionales, cuyas experiencias tienen que ver tanto con circunstancias económicas como con prácticas y orientaciones estéticas bien diferenciadas. No es una sociedad preferentemente de clase media la que se tiene, sino una notablemente polarizada.
No cabe duda que el gráfico A.4 del anexo 2 deja ver que no se estaría ante grupos completamente homogéneos. En ambos bloques ocupacionales es posible identificar diferencias (Gayo, Teitelboim y Méndez, 2013). Entre ellas aparecerían las siguientes: con respecto al grupo ocupacional que manifiesta mayor inactividad cultural, los que participan en menor grado son los menos calificados, tanto los peones del sector primario como los trabajadores no calificados, mientras que aquellos calificados, los empleados de comercio y los trabajadores de los servicios personales, entre otros, se muestran más participativos. En relación con el grupo más activo, se observa de manera destacada que los profesionales tienen un perfil más elitista que los altos ejecutivos, lo que mostraría que la educación es tan importante como el dinero, cuando no más, al menos si se piensa en el activismo cultural o la acumulación de capital cultural en un sentido amplio.

¿Pero qué sucede con los grupos hasta ahora omitidos, esto es, las dueñas de casa, los jubilados, los estudiantes y los desempleados? Normalmente, en las investigaciones no se incorporan estas categorías, y mucho menos con la complejidad que se desarrollan en este trabajo, de modo que poco se sabe con cierto detalle sobre sus patrones y ello se retroalimenta con la escasa reflexión disponible en esta materia. A este respecto, los hallazgos derivados de la presente investigación indican con claridad la necesidad de hacer distinciones dentro de cada uno de estos grupos. Entre las conclusiones más interesantes que se han podido extraer están las siguientes.

i) En todos los casos se observa una fuerte estratificación. Por ejemplo, poco en común parecen tener los jubilados que cuentan con estudios superiores e ingresos mensuales del hogar por sobre $\$ 1.000 .000$ con aquellos que manifiestan tener estudios básicos y un ingreso mensual por debajo de los $\$ 250.000$.

ii) Los estudios parecen ser una variable clave para entender la posición en el espacio social de las categorías de clase que se están analizando. Incluso con ingresos moderados, se observan casos cuyo comportamiento cultural es claramente de tipo participativo. Entre otros, se puede prestar atención a los desempleados con estudios superiores e ingresos entre $\$ 500.000$ y $\$ 1.000 .000$ mensuales, y las dueñas de hogar con estudios superiores e ingresos del hogar dentro de la franja que va de $\$ 250.000$ a $\$ 500.000$. Esto parece indicar que la clave para mostrar un comportamiento participativo es el nivel 
educativo dentro de las condiciones definidas por la disponibilidad de unos recursos económicos mínimos, umbral que parece corresponderse con unos ingresos del hogar entre $\$ 250.000$ y $\$ 500.000$ mensuales.

\section{VI}

\section{Conclusiones}

En este artículo se ha planteado una discusión crítica sobre las características del proceso de terciarización en la sociedad chilena: si bien se registra un aumento de ocupaciones no manuales, y con ello podría pensarse que existe un progresivo avance hacia una sociedad de clase media, hay importantes hallazgos que dificultan aceptar simplemente la afirmación de que Chile se ha convertido en tal tipo de sociedad mesocrática.

En primer lugar, el esperado derrumbe de las fronteras culturales o simbólicas de clase no se ha producido. La evidencia muestra que se vive en sociedades en que, al menos desde el punto de vista cultural, la mayoría de los ciudadanos difícilmente podrían ser calificados de miembros de la clase media. Si esta clase se caracteriza por su activismo cultural, apenas un $17 \%$ de la muestra de encuestados lo representaría adecuadamente, cayendo dicho activismo de forma muy significativa en los otros tipos de perfiles culturales encontrados.

En segundo lugar, lejos de haberse superado el efecto de variables que tradicionalmente se podrían considerar vinculadas al estudio de la desigualdad, la explicación en torno de esta —que se hace evidente a través de las prácticas culturales de los ciudadanosrequiere la combinación de un conjunto amplio de esas variables, entre las que destacan la educación, los recursos económicos y, en menor medida, la edad.

En tercer lugar, en parte como una síntesis de las contribuciones de estas variables, es importante subrayar el papel clave que juega la estructura ocupacional. A este respecto, uno de los patrones que permite entender de mejor manera los hallazgos de este estudio presenta una sociedad polarizada alrededor de dos grandes bloques ocupacionales, que serían "homólogos" 12 o se

12 En la obra de Bourdieu, la tesis de la "homología" significa dos cosas. Por una parte, si se entiende que los campos sociales estarían iii) Se constata que las categorías con ingresos por debajo de los $\$ 250.000$ mensuales y estudios de nivel medio o inferior, formarían parte del mismo conjunto compuesto por las ocupaciones que manifiestan un menor grado de activismo cultural.

corresponderían con dos grandes patrones de participación cultural. Por una parte, estarían las personas con trabajos asociados con remuneraciones y niveles educativos bajos, como serían los casos de diferentes tipos de operarios de la industria y trabajadores de los servicios. Este conjunto de ocupaciones manifestaría muy restringidos niveles de participación cultural. Por otra, se hallarían los trabajos que reciben mayores remuneraciones y suelen exigir niveles educativos más elevados, donde se encontrarían los altos ejecutivos, técnicos de variado tipo y, principalmente, los profesionales.

Finalmente, a modo de síntesis y aceptando que Chile ha experimentado una terciarización parcial de su estructura productiva y ocupacional, una comprensión adecuada de esta sociedad requiere aceptar las siguientes conclusiones. Primero: que la terciarización ha tenido un carácter claramente restringido, cuyos beneficiarios son sobre todo los grupos socioeconómicos altos, que disfrutan de estilos de vida distintivos disponibles para una muy limitada proporción de la población. Segundo: que siguen presentes fundamentos históricos de la desigualdad, como serían los casos del ingreso y la educación, lo que resalta con mucha fuerza en las formas de vivir y manifestarse de las personas. Tercero: que las ocupaciones continúan siendo indicadores centrales de los patrones de comportamiento, y que su perfil cultural denota que Chile es una sociedad dividida tanto económica como culturalmente.

estructurados de acuerdo con una lógica de dominación, ello significaría que los individuos tenderían a estar ubicados en similares posiciones en diferentes campos. Por otra, según esta tesis también existiría una correspondencia entre patrón cultural y estructura social, es decir, la práctica y el gusto culturales estarían estructurados de acuerdo con variables sociales, en especial los capitales económico y cultural. 
ANEXO 1

Actividades estudiadas

CUADRO A. 1

Prácticas culturales analizadas

\begin{tabular}{|c|c|c|c|c|c|c|}
\hline \multirow{2}{*}{ Etiqueta } & \multirow{2}{*}{$\begin{array}{c}\text { Peso relativo } \\
\text { (en porcentajes) }\end{array}$} & \multirow{2}{*}{$\begin{array}{c}\text { Distancia } \\
\text { cuadrática al } \\
\text { origen }\end{array}$} & \multicolumn{2}{|c|}{ Eje 1} & \multicolumn{2}{|c|}{ Eje 2} \\
\hline & & & Coordenadas & Contribuciones & Coordenadas & Contribuciones \\
\hline \multicolumn{7}{|l|}{ Exposiciones } \\
\hline Pintura & 0,333 & 7,593 & 0,99 & 1,86 & $-0,60$ & 1,76 \\
\hline Fotografía & 0,135 & 20,091 & 1,00 & 0,76 & $-0,31$ & 0,20 \\
\hline Otras artes visuales & 0,109 & 25,100 & 1,13 & 0,79 & 0,07 & 0,01 \\
\hline No exposiciones & 2,280 & 0,253 & $-0,26$ & 0,86 & 0,10 & 0,35 \\
\hline \multicolumn{7}{|l|}{ Teatro } \\
\hline Más de 4 veces & 0,111 & 24,778 & 1,42 & 1,26 & $-0,63$ & 0,64 \\
\hline 2 o 3 veces & 0,207 & 12,828 & 1,05 & 1,30 & $-0,77$ & 1,78 \\
\hline Una vez & 0,165 & 16,328 & 0,58 & 0,31 & $-0,22$ & 0,11 \\
\hline No ha asistido & 2,375 & 0,203 & $-0,20$ & 0,53 & 0,11 & 0,43 \\
\hline \multicolumn{7}{|l|}{ Danza } \\
\hline Ballet y danza & 0,132 & 20,637 & 1,11 & 0,93 & $-0,73$ & 1,03 \\
\hline Danza folclórica & 0,509 & 4,613 & 0,44 & 0,55 & $-0,05$ & 0,02 \\
\hline No va a danza & 2,152 & 0,328 & $-0,19$ & 0,46 & 0,05 & 0,07 \\
\hline \multicolumn{7}{|l|}{ Recitales } \\
\hline Música folclórica & 0,157 & 17,236 & 0,55 & 0,26 & $-0,34$ & 0,26 \\
\hline Música rock & 0,265 & 9,791 & 1,03 & 1,60 & $-0,06$ & 0,01 \\
\hline No ha ido a conciertos & 2,044 & 0,398 & $-0,26$ & 0,80 & 0,04 & 0,05 \\
\hline \multicolumn{7}{|l|}{ Pantomima } \\
\hline Sí & 0,263 & 9,847 & 1,28 & 2,46 & 1,59 & 9,80 \\
\hline No & 2,594 & 0,102 & $-0,13$ & 0,25 & $-0,16$ & 1,00 \\
\hline \multicolumn{7}{|l|}{ Malabarismo } \\
\hline Sí & 0,442 & 5,464 & 1,15 & 3,32 & 1,20 & 9,31 \\
\hline No & 2,415 & 0,183 & $-0,21$ & 0,61 & $-0,22$ & 1,70 \\
\hline \multicolumn{7}{|l|}{ Títeres } \\
\hline Sí & 0,207 & 12,782 & 1,51 & 2,69 & 1,59 & 7,73 \\
\hline No & 2,650 & 0,078 & $-0,12$ & 0,21 & $-0,12$ & 0,61 \\
\hline \multicolumn{7}{|l|}{ Magia } \\
\hline Sí & 0,133 & 20,415 & 1,73 & 2,28 & 1,92 & 7,23 \\
\hline No & 2,724 & 0,049 & $-0,08$ & 0,11 & $-0,09$ & 0,35 \\
\hline \multicolumn{7}{|l|}{ Humor } \\
\hline Sí & 0,359 & 6,969 & 1,29 & 3,39 & 1,31 & 8,96 \\
\hline No & 2,499 & 0,143 & $-0,19$ & 0,49 & $-0,19$ & 1,29 \\
\hline \multicolumn{7}{|l|}{ Cine } \\
\hline+7 veces & 0,140 & 19,471 & 1,45 & 1,67 & $-0,56$ & 0,64 \\
\hline De 4 a 6 & 0,178 & 15,062 & 1,00 & 1,02 & $-0,43$ & 0,49 \\
\hline 3 veces & 0,183 & 14,640 & 0,76 & 0,59 & $-0,17$ & 0,08 \\
\hline 2 veces & 0,165 & 16,328 & 0,48 & 0,22 & 0,19 & 0,09 \\
\hline Una vez & 0,138 & 19,673 & 0,42 & 0,14 & $-0,01$ & 0,00 \\
\hline No ha ido al cine & 2,054 & 0,391 & $-0,32$ & 1,19 & 0,08 & 0,18 \\
\hline \multicolumn{7}{|l|}{ Tipo de cine } \\
\hline Acción & 0,844 & 2,384 & $-0,01$ & 0,00 & 0,16 & 0,31 \\
\hline Comedia/humor & 0,472 & 5,052 & 0,01 & 0,00 & $-0,03$ & 0,01 \\
\hline Drama & 0,153 & 17,643 & 0,15 & 0,02 & $-0,36$ & 0,30 \\
\hline Románticas & 0,388 & 6,365 & $-0,26$ & 0,15 & 0,00 & 0,00 \\
\hline Ciencia ficción & 0,200 & 13,253 & 0,39 & 0,18 & $-0,01$ & 0,00 \\
\hline Documental & 0,127 & 21,452 & 0,11 & 0,01 & $-0,62$ & 0,71 \\
\hline Infantiles & 0,118 & 23,279 & 0,25 & 0,04 & 0,07 & 0,01 \\
\hline Suspenso & 0,156 & 17,316 & 0,46 & 0,19 & 0,31 & 0,22 \\
\hline Otro tipo de cine & 0,278 & 9,260 & $-0,68$ & 0,73 & 0,11 & 0,05 \\
\hline
\end{tabular}


Cuadro A.1 (continuación)

\begin{tabular}{|c|c|c|c|c|c|c|}
\hline \multirow{2}{*}{ Etiqueta } & \multirow{2}{*}{$\begin{array}{l}\text { Peso relativo } \\
\text { (en porcentajes) }\end{array}$} & \multirow{2}{*}{$\begin{array}{c}\text { Distancia } \\
\text { cuadrática al } \\
\text { origen }\end{array}$} & \multicolumn{2}{|c|}{ Eje 1} & \multicolumn{2}{|c|}{ Eje 2} \\
\hline & & & Coordenadas & Contribuciones & Coordenadas & Contribuciones \\
\hline \multicolumn{7}{|l|}{ Frecuencia video } \\
\hline $\begin{array}{l}2 \text { o } 3 \text { a la semana DVD } \\
1 \text { DVD a la semana } \\
\text { Cada } 15 \text { días DVD } \\
1-3 \text { meses DVD } \\
\text { Casi nunca DVD } \\
\text { No ve videos }\end{array}$ & $\begin{array}{l}0,595 \\
0,536 \\
0,305 \\
0,508 \\
0,166 \\
0,746\end{array}$ & $\begin{array}{r}3,800 \\
4,333 \\
8,363 \\
4,620 \\
16,185 \\
2,828\end{array}$ & $\begin{array}{r}0,54 \\
0,25 \\
0,17 \\
0,06 \\
-0,30 \\
-0,65\end{array}$ & $\begin{array}{l}0,97 \\
0,18 \\
0,05 \\
0,01 \\
0,08 \\
1,77\end{array}$ & $\begin{array}{r}0,13 \\
-0,01 \\
-0,05 \\
-0,13 \\
0,01 \\
0,00\end{array}$ & $\begin{array}{l}0,15 \\
0,00 \\
0,01 \\
0,12 \\
0,00 \\
0,00\end{array}$ \\
\hline \multicolumn{7}{|l|}{ Frecuencia libros } \\
\hline $\begin{array}{l}\text { Libros todos los día } \\
\text { Libros cada } 15 \text { días } \\
1-3 \text { meses } 1 \text { libro } \\
\text { Libro } 1 \text { al año } \\
\text { No lee libros }\end{array}$ & $\begin{array}{l}0,398 \\
0,249 \\
0,272 \\
0,226 \\
1,711\end{array}$ & $\begin{array}{r}6,175 \\
10,473 \\
9,492 \\
11,616 \\
0,670\end{array}$ & $\begin{array}{r}0,58 \\
0,56 \\
0,80 \\
0,43 \\
-0,40\end{array}$ & $\begin{array}{l}0,77 \\
0,44 \\
0,99 \\
0,24 \\
1,56\end{array}$ & $\begin{array}{r}-0,83 \\
-0,43 \\
-0,29 \\
0,08 \\
0,29\end{array}$ & $\begin{array}{l}4,03 \\
0,66 \\
0,34 \\
0,02 \\
2,13\end{array}$ \\
\hline \multicolumn{7}{|l|}{ Tipo de libros } \\
\hline $\begin{array}{l}\text { Novelas } \\
\text { Cuentos } \\
\text { Libros de autoayuda } \\
\text { Historia y biografía } \\
\text { Tecnología, ciencias } \\
\text { Religiosos } \\
\text { Otros } \\
\text { Ninguno, no lee }\end{array}$ & $\begin{array}{l}0,813 \\
0,272 \\
0,190 \\
0,213 \\
0,112 \\
0,207 \\
0,248 \\
0,597\end{array}$ & $\begin{array}{r}2,512 \\
9,519 \\
14,076 \\
12,428 \\
24,463 \\
12,828 \\
10,536 \\
3,789\end{array}$ & $\begin{array}{r}0,24 \\
0,09 \\
0,51 \\
0,28 \\
0,54 \\
-0,44 \\
-0,17 \\
-0,62\end{array}$ & $\begin{array}{l}0,26 \\
0,01 \\
0,28 \\
0,09 \\
0,19 \\
0,23 \\
0,04 \\
1,30\end{array}$ & $\begin{array}{r}-0,21 \\
0,22 \\
-0,42 \\
-0,25 \\
-0,32 \\
-0,32 \\
0,04 \\
0,56\end{array}$ & $\begin{array}{l}0,53 \\
0,20 \\
0,49 \\
0,19 \\
0,17 \\
0,32 \\
0,01 \\
2,77\end{array}$ \\
\hline \multicolumn{7}{|l|}{ Museo } \\
\hline $\begin{array}{l}\text { Museo de arte } \\
\text { Museo histórico } \\
\text { No va al museo }\end{array}$ & $\begin{array}{l}0,120 \\
0,288 \\
2,285\end{array}$ & $\begin{array}{r}22,727 \\
8,919 \\
0,250\end{array}$ & $\begin{array}{r}1,46 \\
0,82 \\
-0,24\end{array}$ & $\begin{array}{l}1,46 \\
1,09 \\
0,72\end{array}$ & $\begin{array}{r}-0,74 \\
-0,12 \\
0,06\end{array}$ & $\begin{array}{l}0,97 \\
0,06 \\
0,12\end{array}$ \\
\hline \multicolumn{7}{|l|}{ Frecuencia TV } \\
\hline $\begin{array}{l}\text { Todos los días } \\
\text { Casi todos los dias } \\
1 \text { a } 3 \text { días a la sema }\end{array}$ & $\begin{array}{l}2,286 \\
0,293 \\
0,228\end{array}$ & $\begin{array}{r}0,250 \\
8,757 \\
11,541\end{array}$ & $\begin{array}{r}-0,02 \\
0,14 \\
0,06\end{array}$ & $\begin{array}{l}0,01 \\
0,03 \\
0,00\end{array}$ & $\begin{array}{r}0,06 \\
-0,29 \\
-0,22\end{array}$ & $\begin{array}{l}0,14 \\
0,35 \\
0,15\end{array}$ \\
\hline \multicolumn{7}{|l|}{ Artesanía } \\
\hline $\begin{array}{l}\text { Sí } \\
\text { No }\end{array}$ & $\begin{array}{l}1,638 \\
1,219\end{array}$ & $\begin{array}{l}0,744 \\
1,343\end{array}$ & $\begin{array}{r}0,34 \\
-0,45\end{array}$ & $\begin{array}{l}1,05 \\
1,41\end{array}$ & $\begin{array}{r}-0,08 \\
0,11\end{array}$ & $\begin{array}{l}0,15 \\
0,20\end{array}$ \\
\hline \multicolumn{7}{|l|}{ Pinturas originales } \\
\hline $\begin{array}{l}\text { Sí } \\
\text { No }\end{array}$ & $\begin{array}{l}0,406 \\
2,451\end{array}$ & $\begin{array}{l}6,030 \\
0,166\end{array}$ & $\begin{array}{r}1,02 \\
-0,17\end{array}$ & $\begin{array}{l}2,42 \\
0,40\end{array}$ & $\begin{array}{r}-0,39 \\
0,06\end{array}$ & $\begin{array}{l}0,90 \\
0,15\end{array}$ \\
\hline \multicolumn{7}{|l|}{ Esculturas } \\
\hline $\begin{array}{l}\text { Sí } \\
\text { No }\end{array}$ & $\begin{array}{l}0,183 \\
2,674\end{array}$ & $\begin{array}{r}14,640 \\
0,068\end{array}$ & $\begin{array}{r}1,36 \\
-0,09\end{array}$ & $\begin{array}{l}1,92 \\
0,13\end{array}$ & $\begin{array}{r}-0,50 \\
0,03\end{array}$ & $\begin{array}{l}0,68 \\
0,05\end{array}$ \\
\hline \multicolumn{7}{|l|}{ Grabados } \\
\hline $\begin{array}{l}\text { Sí } \\
\text { No }\end{array}$ & $\begin{array}{l}0,197 \\
2,660\end{array}$ & $\begin{array}{r}13,500 \\
0,074\end{array}$ & $\begin{array}{r}1,29 \\
-0,10\end{array}$ & $\begin{array}{l}1,87 \\
0,14\end{array}$ & $\begin{array}{r}-0,40 \\
0,03\end{array}$ & $\begin{array}{l}0,46 \\
0,03\end{array}$ \\
\hline \multicolumn{7}{|l|}{ Libros de colección } \\
\hline $\begin{array}{l}\text { Sí } \\
\text { No }\end{array}$ & $\begin{array}{l}0,363 \\
2,495\end{array}$ & $\begin{array}{l}6,879 \\
0,145\end{array}$ & $\begin{array}{r}1,15 \\
-0,17\end{array}$ & $\begin{array}{l}2,71 \\
0,39\end{array}$ & $\begin{array}{r}-0,46 \\
0,07\end{array}$ & $\begin{array}{l}1,12 \\
0,16\end{array}$ \\
\hline \multicolumn{7}{|l|}{ Sección diarios } \\
\hline $\begin{array}{l}\text { Noticias internacionales } \\
\text { Nacional crónica } \\
\text { Nacional política } \\
\text { Nacional policial } \\
\text { Deportiva } \\
\text { Páginas sociales } \\
\text { Lo lee completo } \\
\text { No lee el diario }\end{array}$ & $\begin{array}{l}0,187 \\
0,289 \\
0,119 \\
0,259 \\
0,200 \\
0,124 \\
0,466 \\
0,863\end{array}$ & $\begin{array}{r}14,297 \\
8,896 \\
23,000 \\
10,048 \\
13,301 \\
22,072 \\
5,132 \\
2,309\end{array}$ & $\begin{array}{r}0,21 \\
0,15 \\
0,36 \\
0,07 \\
0,14 \\
0,14 \\
0,27 \\
-0,50\end{array}$ & $\begin{array}{l}0,05 \\
0,04 \\
0,09 \\
0,01 \\
0,02 \\
0,01 \\
0,19 \\
1,25\end{array}$ & $\begin{array}{r}-0,63 \\
-0,05 \\
-0,36 \\
0,18 \\
0,48 \\
0,31 \\
-0,24 \\
0,20\end{array}$ & $\begin{array}{l}1,07 \\
0,01 \\
0,22 \\
0,12 \\
0,68 \\
0,18 \\
0,40 \\
0,50\end{array}$ \\
\hline
\end{tabular}


Cuadro A.1 (conclusión)

\begin{tabular}{|c|c|c|c|c|c|c|}
\hline \multirow{2}{*}{ Etiqueta } & \multirow{2}{*}{$\begin{array}{c}\text { Peso relativo } \\
\text { (en porcentajes) }\end{array}$} & \multirow{2}{*}{$\begin{array}{c}\text { Distancia } \\
\text { cuadrática al } \\
\text { origen }\end{array}$} & \multicolumn{2}{|c|}{ Eje 1} & \multicolumn{2}{|c|}{ Eje 2} \\
\hline & & & Coordenadas & Contribuciones & Coordenadas & Contribuciones \\
\hline \multicolumn{7}{|l|}{ Programa radio } \\
\hline $\begin{array}{l}\text { Música } \\
\text { Noticias } \\
\text { Pro opinión radio } \\
\text { No escucha radio }\end{array}$ & $\begin{array}{l}1,808 \\
0,436 \\
0,135 \\
0,304\end{array}$ & $\begin{array}{r}0,581 \\
5,556 \\
20,198 \\
8,384\end{array}$ & $\begin{array}{r}0,01 \\
-0,11 \\
0,22 \\
0,07\end{array}$ & $\begin{array}{l}0,00 \\
0,03 \\
0,04 \\
0,01\end{array}$ & $\begin{array}{r}0,05 \\
-0,31 \\
0,01 \\
0,11\end{array}$ & $\begin{array}{l}0,06 \\
0,60 \\
0,00 \\
0,05\end{array}$ \\
\hline \multicolumn{7}{|l|}{ Tipo revistas } \\
\hline $\begin{array}{l}\text { Diseño y decoración } \\
\text { Deportes } \\
\text { Actualidad (política) } \\
\text { Variedades (revistas) } \\
\text { Otra } \\
\text { No lee revistas }\end{array}$ & $\begin{array}{l}0,129 \\
0,138 \\
0,187 \\
0,363 \\
0,183 \\
1,608\end{array}$ & $\begin{array}{r}21,213 \\
19,776 \\
14,241 \\
6,879 \\
14,582 \\
0,777\end{array}$ & $\begin{array}{r}0,43 \\
0,59 \\
0,71 \\
0,45 \\
0,32 \\
-0,40\end{array}$ & $\begin{array}{l}0,14 \\
0,27 \\
0,54 \\
0,42 \\
0,11 \\
1,48\end{array}$ & $\begin{array}{r}-0,48 \\
0,49 \\
-0,74 \\
0,05 \\
-0,24 \\
0,15\end{array}$ & $\begin{array}{l}0,44 \\
0,48 \\
1,52 \\
0,01 \\
0,16 \\
0,52\end{array}$ \\
\hline \multicolumn{7}{|l|}{ Uso Internet } \\
\hline $\begin{array}{l}\text { Comunicarse por Internet } \\
\text { Buscar información Internet } \\
\text { Bajar música Internet } \\
\text { Otro Internet } \\
\text { No usa Internet }\end{array}$ & $\begin{array}{l}0,694 \\
0,436 \\
0,122 \\
0,193 \\
1,413\end{array}$ & $\begin{array}{r}3,118 \\
5,556 \\
22,461 \\
13,809 \\
1,022\end{array}$ & $\begin{array}{r}0,54 \\
0,54 \\
0,43 \\
0,62 \\
-0,55\end{array}$ & $\begin{array}{l}1,16 \\
0,72 \\
0,13 \\
0,42 \\
2,47\end{array}$ & $\begin{array}{r}0,03 \\
-0,46 \\
0,31 \\
-0,05 \\
0,11\end{array}$ & $\begin{array}{l}0,01 \\
1,33 \\
0,17 \\
0,01 \\
0,24\end{array}$ \\
\hline \multicolumn{7}{|l|}{$\mathrm{N}^{\mathrm{o}}$ de libros } \\
\hline $\begin{array}{l}\text { No tiene libros } \\
\text { Entre } 1-10 \text { libros } \\
\text { Entre } 11 \text { y } 25 \text { libros } \\
\text { Entre } 26 \text { y } 50 \text { libros } \\
\text { Entre } 51 \text { y } 100 \text { libro } \\
\text { Más de } 100 \text { libros } \\
\text { ns/nr libros }\end{array}$ & $\begin{array}{l}0,445 \\
0,838 \\
0,501 \\
0,459 \\
0,309 \\
0,229 \\
0,076\end{array}$ & $\begin{array}{r}5,425 \\
2,409 \\
4,705 \\
5,224 \\
8,239 \\
11,466 \\
36,622\end{array}$ & $\begin{array}{r}-0,89 \\
-0,34 \\
0,12 \\
0,34 \\
0,67 \\
1,24 \\
-0,39\end{array}$ & $\begin{array}{l}1,99 \\
0,55 \\
0,04 \\
0,31 \\
0,78 \\
2,00 \\
0,06\end{array}$ & $\begin{array}{r}0,47 \\
0,13 \\
0,15 \\
-0,25 \\
-0,38 \\
-0,76 \\
0,20\end{array}$ & $\begin{array}{l}1,46 \\
0,20 \\
0,16 \\
0,42 \\
0,66 \\
1,95 \\
0,04\end{array}$ \\
\hline \multicolumn{7}{|l|}{ Viaje fuera del país } \\
\hline $\begin{array}{l}\text { Sí } \\
\text { No }\end{array}$ & $\begin{array}{l}0,185 \\
2,672\end{array}$ & $\begin{array}{r}14,410 \\
0,069\end{array}$ & $\begin{array}{r}1,15 \\
-0,08\end{array}$ & $\begin{array}{l}1,39 \\
0,10\end{array}$ & $\begin{array}{r}-0,46 \\
0,03\end{array}$ & $\begin{array}{l}0,57 \\
0,04\end{array}$ \\
\hline \multicolumn{7}{|l|}{ Viaje dentro del país } \\
\hline $\begin{array}{l}\text { Sí } \\
\text { No }\end{array}$ & $\begin{array}{l}0,923 \\
1,934\end{array}$ & $\begin{array}{l}2,096 \\
0,477\end{array}$ & $\begin{array}{r}0,72 \\
-0,34\end{array}$ & $\begin{array}{l}2,73 \\
1,30\end{array}$ & $\begin{array}{r}0,05 \\
-0,02\end{array}$ & $\begin{array}{l}0,04 \\
0,02\end{array}$ \\
\hline \multicolumn{7}{|l|}{ Ir al estadio } \\
\hline $\begin{array}{l}\text { Sí } \\
\text { No }\end{array}$ & $\begin{array}{l}0,373 \\
2,484\end{array}$ & $\begin{array}{l}6,662 \\
0,150\end{array}$ & $\begin{array}{r}0,63 \\
-0,10\end{array}$ & $\begin{array}{l}0,85 \\
0,13\end{array}$ & $\begin{array}{r}0,48 \\
-0,07\end{array}$ & $\begin{array}{l}1,27 \\
0,19\end{array}$ \\
\hline \multicolumn{7}{|l|}{ Visita sitios patrimoniales } \\
\hline $\begin{array}{l}\text { Sí } \\
\text { No }\end{array}$ & $\begin{array}{l}0,275 \\
2,582\end{array}$ & $\begin{array}{l}9,388 \\
0,107\end{array}$ & $\begin{array}{r}1,61 \\
-0,17\end{array}$ & $\begin{array}{l}4,06 \\
0,43\end{array}$ & $\begin{array}{r}-0,09 \\
0,01\end{array}$ & $\begin{array}{l}0,03 \\
0,00\end{array}$ \\
\hline \multicolumn{7}{|l|}{ Ferias de artesanía } \\
\hline $\begin{array}{l}\text { Sí } \\
\text { No }\end{array}$ & $\begin{array}{l}0,762 \\
2,095\end{array}$ & $\begin{array}{l}2,749 \\
0,364\end{array}$ & $\begin{array}{r}0,79 \\
-0,29\end{array}$ & $\begin{array}{l}2,70 \\
0,98\end{array}$ & $\begin{array}{r}0,13 \\
-0,05\end{array}$ & $\begin{array}{l}0,17 \\
0,06\end{array}$ \\
\hline \multicolumn{7}{|l|}{ Conferencias } \\
\hline $\begin{array}{l}\text { Sí } \\
\text { No }\end{array}$ & $\begin{array}{l}0,167 \\
2,690\end{array}$ & $\begin{array}{r}16,115 \\
0,062\end{array}$ & $\begin{array}{r}1,77 \\
-0,11\end{array}$ & $\begin{array}{l}2,95 \\
0,18\end{array}$ & $\begin{array}{r}-0,39 \\
0,02\end{array}$ & $\begin{array}{l}0,36 \\
0,02\end{array}$ \\
\hline \multicolumn{7}{|l|}{ Parque diversiones } \\
\hline $\begin{array}{l}\text { Sí } \\
\text { No }\end{array}$ & $\begin{array}{l}0,372 \\
2,485\end{array}$ & $\begin{array}{l}6,676 \\
0,150\end{array}$ & $\begin{array}{r}1,10 \\
-0,16\end{array}$ & $\begin{array}{l}2,53 \\
0,38\end{array}$ & $\begin{array}{r}0,79 \\
-0,12\end{array}$ & $\begin{array}{l}3,43 \\
0,51\end{array}$ \\
\hline \multicolumn{7}{|l|}{ Zoológico } \\
\hline $\begin{array}{l}\text { Sí } \\
\text { No }\end{array}$ & $\begin{array}{l}0,178 \\
2,679\end{array}$ & $\begin{array}{r}15,062 \\
0,066\end{array}$ & $\begin{array}{r}1,41 \\
-0,09\end{array}$ & $\begin{array}{l}2,02 \\
0,13\end{array}$ & $\begin{array}{r}0,84 \\
-0,06\end{array}$ & $\begin{array}{l}1,84 \\
0,12\end{array}$ \\
\hline \multicolumn{7}{|l|}{ Actividades deportivas } \\
\hline $\begin{array}{l}\text { Sí } \\
\text { No }\end{array}$ & $\begin{array}{l}0,464 \\
2,393\end{array}$ & $\begin{array}{l}5,159 \\
0,194\end{array}$ & $\begin{array}{r}0,87 \\
-0,17\end{array}$ & $\begin{array}{l}1,98 \\
0,38\end{array}$ & $\begin{array}{r}0,50 \\
-0,10\end{array}$ & $\begin{array}{l}1,69 \\
0,33\end{array}$ \\
\hline
\end{tabular}

Fuente: Elaboración propia sobre la base de datos de la Encuesta de Participación y Consumo Cultural. 
ANEXO 2

Categorías de clase

CUADRO A. 1

Clasificación de categorías de clase empleada en el análisis

\begin{tabular}{|c|c|c|}
\hline Actividad u ocupación & Frecuencia & Porcentaje \\
\hline Altos ejecutivos & 32 & 0,78 \\
\hline Profesionales & 225 & 5,39 \\
\hline Técnicos en ingeniería, salud y otros & 56 & 1,33 \\
\hline Profesores de enseñanza básica & 9 & 0,23 \\
\hline Otros técnicos y profesionales de nivel medio & 120 & 2,88 \\
\hline Empleados de oficina & 109 & 2,61 \\
\hline Empleados que atienden clientes & 58 & 1,38 \\
\hline Trabajador de los servicios personales & 142 & 3,40 \\
\hline Empleados de comercio & 269 & 6,45 \\
\hline Trabajadores calificados en agricultura y pesca & 22 & 0,52 \\
\hline Operarios industria de extracción y construcción & 148 & 3,55 \\
\hline Operarios industria metalúrgica y mecánica & 91 & 2,17 \\
\hline Mecánicos de precisión y artesanos & 116 & 2,78 \\
\hline Operarios maquinaria & 49 & 1,17 \\
\hline Conductores & 149 & 3,56 \\
\hline Trabajadores no calificados ventas y servicios & 174 & 4,17 \\
\hline Peones sector primario y secundario & 150 & 3,59 \\
\hline Ocupado s/o & 33 & 0,80 \\
\hline DES-BASI-<250 & 29 & 0,69 \\
\hline DES-MED<250 & 45 & 1,09 \\
\hline DES-ME 250-500 & 21 & 0,51 \\
\hline DES-U-250-500 & 22 & 0,54 \\
\hline DES-ME - 500 A 1 & 24 & 0,57 \\
\hline DES-SU 500 A 1M & 25 & 0,59 \\
\hline HOG-BAS - <250 & 193 & 4,62 \\
\hline HOG-MED <250 & 168 & 4,03 \\
\hline HOG- ECPR $<250$ & 41 & 0,97 \\
\hline HO-BAS-250-500 & 35 & 0,85 \\
\hline HOG-MED-250-50 & 150 & 3,59 \\
\hline HOG-TECP250 A 500 & 28 & 0,68 \\
\hline HOG-SUPPR-250 A 500 & 22 & 0,54 \\
\hline HOG-MED500 A 1 & 22 & 0,54 \\
\hline EST-MEDIA $<250$ & 54 & 1,30 \\
\hline EST-MEDIA 250 A 500 & 64 & 1,54 \\
\hline EST-TECPR 250 A 500 & 22 & 0,52 \\
\hline EST-SUPU 250 A 500 & 39 & 0,94 \\
\hline EST-MED 500 A 1 M & 31 & 0,75 \\
\hline EST-SUPUN 500-1M & 37 & 0,87 \\
\hline EST-SUPUN+1 MILL & 57 & 1,37 \\
\hline JUB. BAS $<250$ & 131 & 3,15 \\
\hline JUB. MED < 250 & 65 & 1,55 \\
\hline JUB. BAS 250 A 500 & 26 & 0,62 \\
\hline JUB. SUP UNIV+1M & 30 & 0,73 \\
\hline OTROS NO OCUP & 840 & 20,11 \\
\hline Total & 4176 & 100,00 \\
\hline
\end{tabular}

Fuente: Elaboración propia sobre la base de datos de la Encuesta de Participación y Consumo Cultural.

Nota: OTROS NO OCUP: Otros no ocupados; DES: Desocupado; HOG: Quehaceres del hogar; EST: Estudiante; JUB: Jubilado;

BAS: Educación básica; MED: Educación media; TECPR: Educación técnica profesional; SUP UNIV: Educación superior universitaria; < 250: Ingresos del hogar menores a \$250.000; 250A500: Ingresos del hogar entre \$250.000 y \$500.000; 500A1M: Ingresos del hogar entre $\$ 500.000$ y $\$ 1.000 .000 ;+1 \mathrm{M}$ : Ingresos superiores a $\$ 1.000 .000$. 
CUADRO A.2

Tipos de práctica cultural

\begin{tabular}{|c|c|c|}
\hline & Joven & Adulto \\
\hline Bajo estatus $^{a}$ & \multicolumn{2}{|c|}{ Escasa participación cultural } \\
\hline Alto estatus & $\begin{array}{l}\text { Cultura juvenil } \\
\text { Alto activismo }\end{array}$ & $\begin{array}{l}\text { Cultura elitista } \\
\text { Alto activismo }\end{array}$ \\
\hline
\end{tabular}

Fuente: Elaboración propia sobre la base de datos de la Encuesta de Participación y Consumo Cultural.

a Por "estatus" aquí solo se hace referencia a la acumulación de los recursos económicos y educativos.

GRÁFICO A.1

Espacio social de la práctica y el gusto culturales

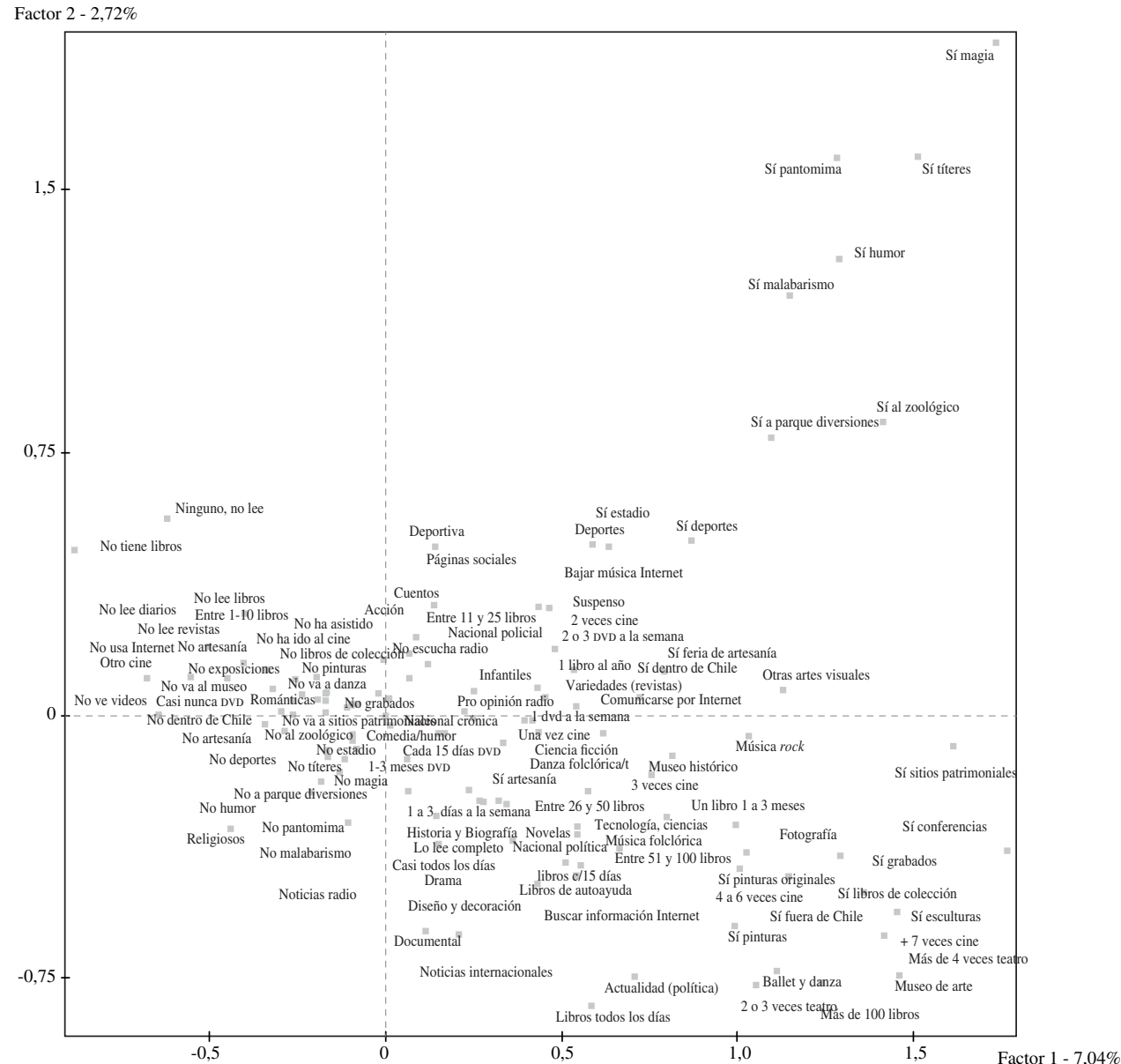

Fuente: Elaboración propia sobre la base de datos de la Encuesta de Participación y Consumo Cultural. 
GRÁFICO A.2

Variables sociodemográficas que estructuran el espacio sociocultural

(Lineas de trayectoria para nivel educativo, grupo socioeconómico y edad)

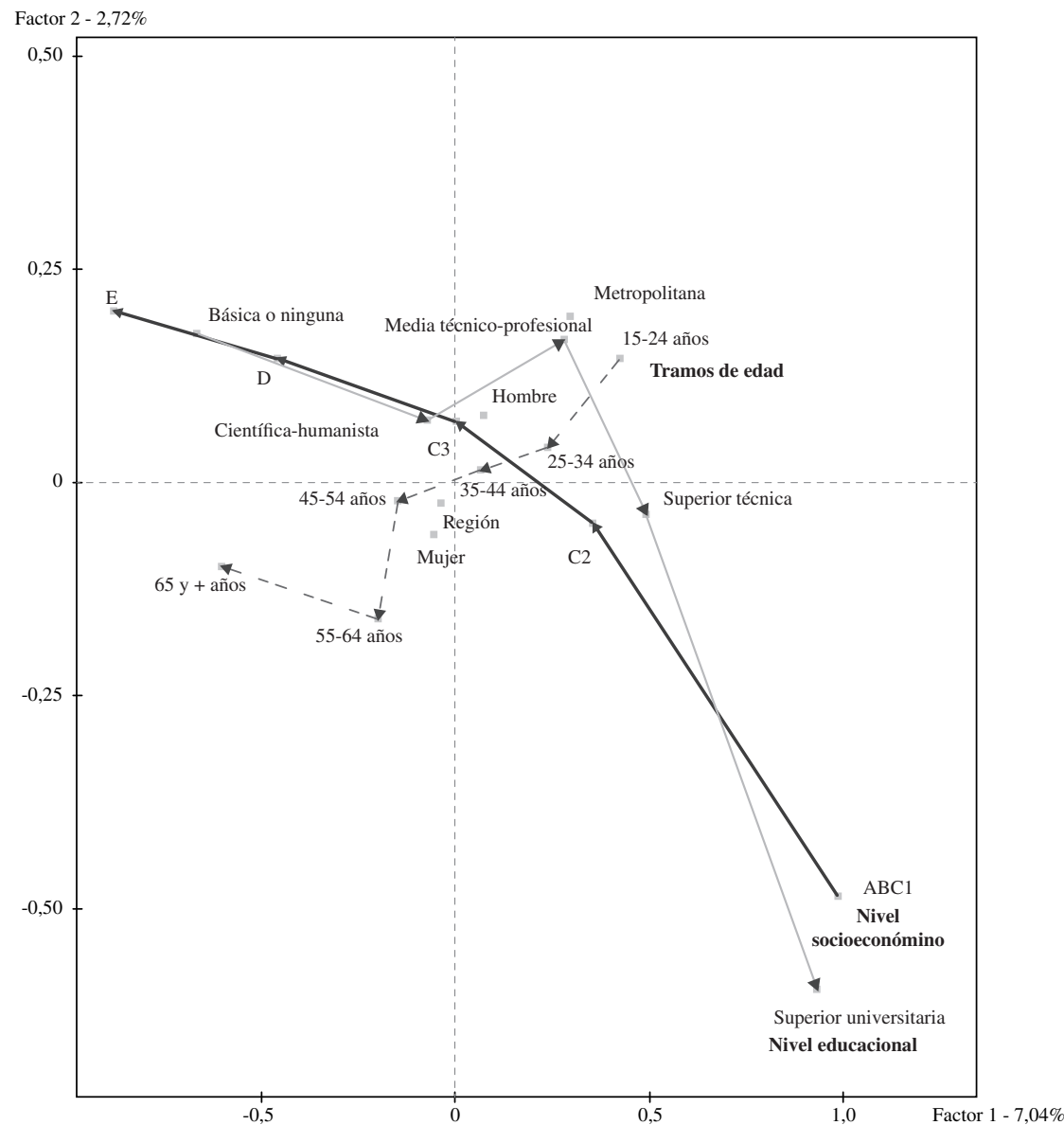

Fuente: Elaboración propia sobre la base de datos de la Encuesta de Participación y Consumo Cultural. 
GRÁFICO A.3

Representación espacial de la tipología de consumidores culturales

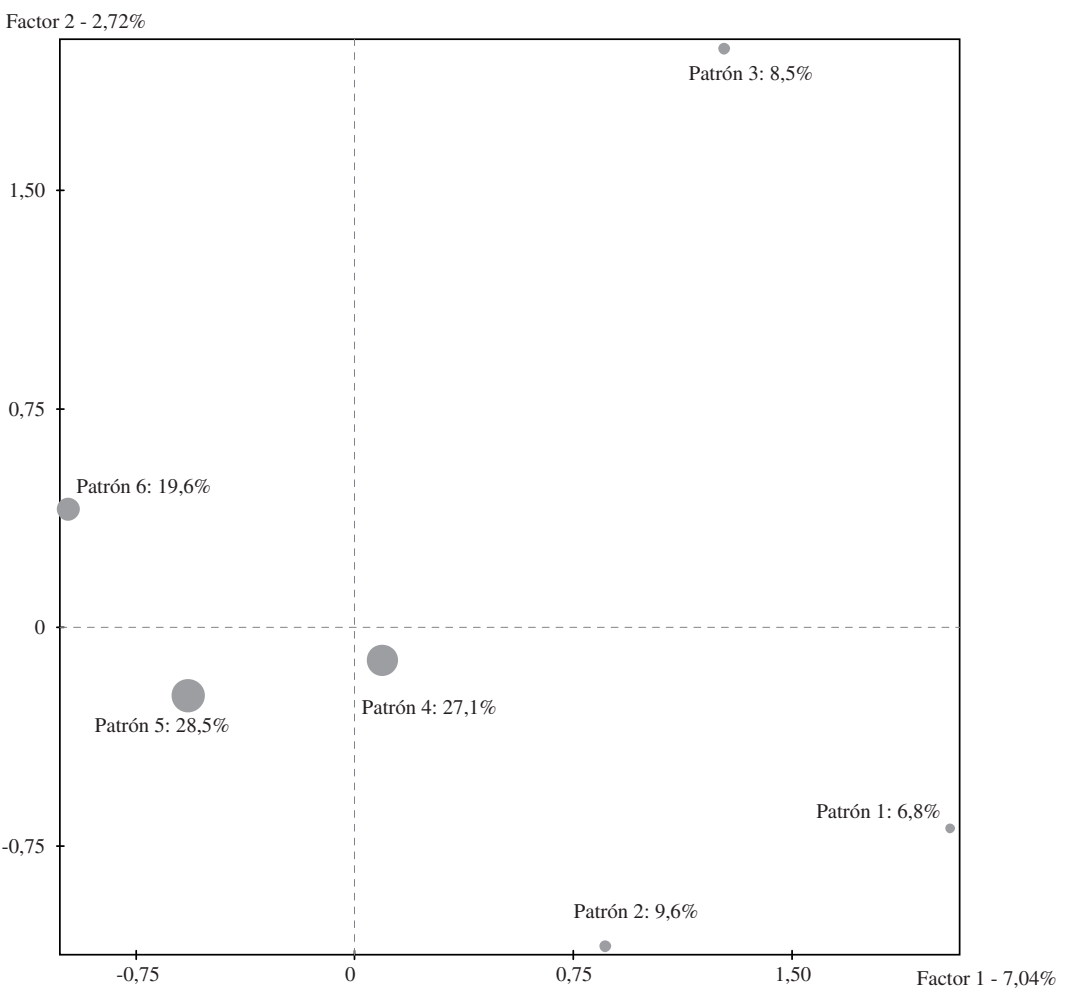

Fuente: Elaboración propia sobre la base de datos de la Encuesta de Participación y Consumo Cultural. 
GRÁFICO A.4

Variables que estructuran el espacio sociocultural: dimensión ocupacional

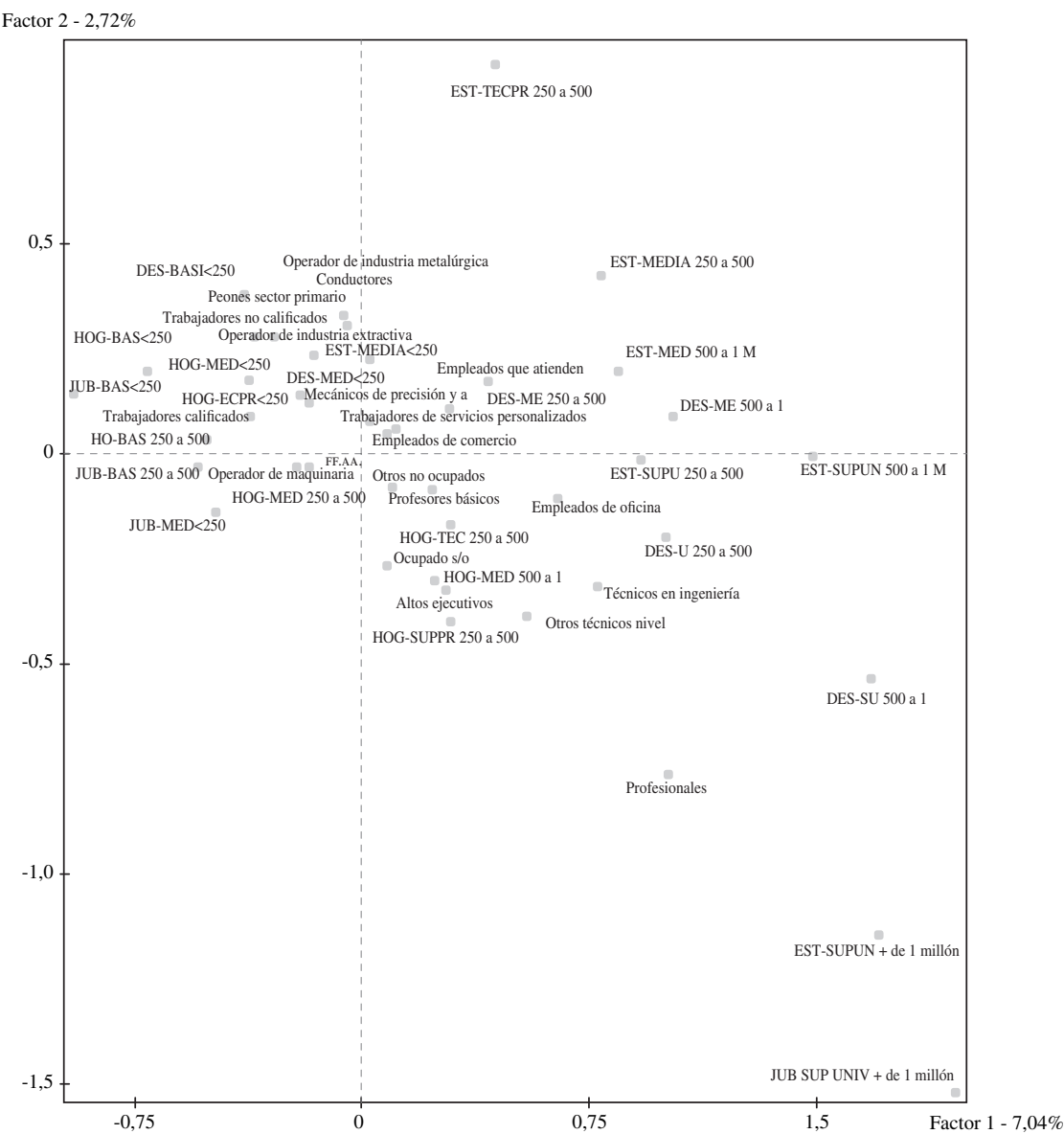

Fuente: Elaboración propia sobre la base de datos de la Encuesta de Participación y Consumo Cultural.

Nota: DES: Desocupado; HOG: Quehaceres del hogar; EST: Estudiante; JUB: Jubilado; BAS: Educación básica; MED: Educación media; TECPR: Educación técnico profesional; SUPUN: Educación superior universitaria; < 250: Ingresos del hogar menores de \$250.000; 250 a 500: Ingresos del hogar entre $\$ 250.000$ y $\$ 500.000 ; 500$ a $1 \mathrm{M}$ : Ingresos del hogar entre $\$ 500.000$ y $\$ 1.000 .000 ;+1 \mathrm{M}$ : Ingresos superiores a $\$ 1.000 .000$. 


\section{Bibliografía}

Aguilar, O. (2009), "Principios de diferenciación material y simbólica en la estratificación social", El arte de clasificar a los chilenos. Enfoques sobre los modelos de estratificación en Chile, A. Joignant y P. Güell (coords.), Santiago, Ediciones Universidad Diego Portales.

Allen, J. (1992), "Post-industrialism and post-Fordism", Modernity and Its Futures, S. Hall, D. Held y T. McGrew (eds.), Cambridge, Polity Press/Open University.

Atria, R. (2004), "Estructura ocupacional, estructura social y clases sociales", serie Politicas Sociales, No 96 (LC/L.2192-P), Santiago, Comisión Económica para América Latina y el Caribe (CEPAL)

Atria, R., R. Franco y A. León (coords.) (2007), Estratificación y movilidad social en América Latina. Transformaciones estructurales de un cuarto de siglo, Santiago, Lom Ediciones/ Comisión Económica para América Latina y el Caribe (CEPAL).

Beck, U. y E. Beck-Gernsheim (2002), Individualization, Londres, SAGE.

Bell, D. (1974), The Coming of Post-Industrial Society. A Venture in Social Forecasting, Londres, Heinemann.

Bennett, T. y otros (2009), Culture, Class, Distinction, Londres, Routledge.

Bennett, T., M. Bustamante y J. Frow (2013), "The Australian space of lifestyles in comparative perspective", Journal of Sociology, vol. 49, $\mathrm{N}^{\circ} 2-3$, SAGE.

Bernasconi, O. y E. Puentes (2001), Gasto en bienes y servicios culturales de los hogares del Gran Santiago: 1988-1997, Santiago, Ministerio de Desarrollo Social.

Bottomore, T. y P. Goode (eds.) (1978), Austro-Marxism, Oxford, Clarendon Press.

Bourdieu, P. (1979), La distinction, París, Les Éditions de Minuit.

Brunner, J.J., A. Barrios y C. Catalán (1989), Chile: transformaciones culturales y modernidad, Santiago, Facultad Latinoamericana de Ciencias Sociales (FLACSO).

Canales, M. (2007), "Ni pobres ni incluidos: ¿nueva cuestión social?”, Revista de Sociología, № 21, Santiago, Universidad de Chile.

Carabaña, J. y A. Francisco (comps.) (1994), Teorías contemporáneas de las clases sociales, Madrid, Editorial Pablo Iglesias.

Catalán, C. (2009), "Consumo y segmentación: algunas consideraciones conceptuales y empíricas", El arte de clasificar a los chilenos. Enfoques sobre los modelos de estratificación en Chile, A. Joignant y P. Güell (coords.), Santiago, Ediciones Universidad Diego Portales.

Catalán, C. y G. Sunkel (1990), "Consumo cultural en Chile: la elite, lo masivo y lo popular", Documento de Trabajo, $\mathrm{N}^{\circ} 455$, Santiago, Facultad Latinoamericana de Ciencias Sociales (FLACSO).

Catalán, C. y P. Torche (2005), Consumo cultural en Chile: miradas y perspectivas, Santiago, Instituto Nacional de Estadísticas/ Consejo Nacional de la Cultura y las Artes.

CNCA (Consejo Nacional de la Cultura y las Artes) (2011a), Segunda Encuesta Nacional de Participación y Consumo Cultural (ENPCC), Valparaíso, Ediciones Cultura.

(2011b), Política cultural 2011-2016, Valparaíso.

(2007), Encuesta de Consumo Cultural 2004-2005, Valparaíso

Daude, C. (2012), "Educación, clases medias y movilidad social en América Latina", Las clases medias en sociedades desiguales, Pensamiento Iberoamericano, $\mathrm{N}^{\circ} 10$.

Délano Urrutia, M. (2011), "Las políticas culturales en Chile como motor del desarrollo humano", Políticas culturales: contingencia y desafíos, Santiago, LOM Ediciones.

El Mercurio (2009), "Más de la mitad de la población no participa en actividades culturales", Santiago, 8 de enero.

Erikson, R. y J.H. Goldthorpe (1993), The Constant Flux. A Study of Class Mobility in Industrial Societies, Oxford, Clarendon Press.
Espinoza, V. y E. Barozet (2009), “¿De qué hablamos cuando decimos "clase media"'? Perspectivas sobre el caso chileno", El arte de clasificar a los chilenos. Enfoques sobre los modelos de estratificación en Chile, A. Joignant y P. Güell (coords.), Santiago, Ediciones Universidad Diego Portales.

Ferreira, F. y otros (2013), Economic Mobility and the Rise of the Latin American Middle Class, Washington, D.C., Banco Mundial.

Filgueira, C. (2001), "La actualidad de viejas temáticas: sobre los estudios de clase, estratificación y movilidad social en América Latina", serie Políticas Sociales, $\mathrm{N}^{\circ} 51$ (LC/L.1582-P), Santiago, Comisión Económica para América Latina y el Caribe (CEPAL).

Franco, R., M. Hopenhayn y A. León (coords.) (2010), Las clases medias en América Latina, Santiago, Ciudad de México, Siglo xxi/Comisión Económica para América Latina y el Caribe (CEPAL).

Galbraith, J.K. (1967), The New Industrial State, Princeton, Princeton University Press.

Gayo, M. (2013a), "Revisiting middle-class politics: a multidimensional approach - evidence from Spain", The Sociological Review, vol. 61, $\mathrm{N}^{\circ} 4$, Wiley.

(2013b), "La teoría del capital cultural y la participación de los jóvenes. El caso chileno como ejemplo", Última Década, $\mathrm{N}^{\circ} 38$, Valparaíso, Centro de Estudios Sociales CIDPA.

(2013c), "El problema de la delimitación o boundary problem. Una aproximación a la definición de la clase media", Polis. Revista Latinoamericana, vol. 12, $\mathrm{N}^{\circ} 36$.

(2008), “'Sigue vivo el sector de empleo? La fractura entre sectores de empleo y el comportamiento político en la clase de servicio: España, 1989-2003”, Administración y Ciudadanía, vol. 3, $\mathrm{N}^{\circ} 2$, Escuela Gallega de Administración Pública.

(2006), "Leisure and participation in Britain", Cultural Trends, vol. 15, $\mathrm{N}^{\circ} 2-3$, Taylor \& Francis.

(2003), "Divisiones de sector de empleo y comportamiento político en la clase de servicio: España, 1989-2000", Revista Internacional de Sociología, vol. 61, $\mathrm{N}^{\circ} 35$.

Gayo, M., M. Savage y A. Warde (2006), "A cultural map of the United Kingdom, 2003", Cultural Trends, 58/59, vol. 15, $\mathrm{N}^{\circ}$ 2-3, Taylor \& Francis.

Gayo, M. y otros (2011), "Consumo cultural y desigualdad de clase, género y edad: un estudio comparado en Argentina, Chile y Uruguay", serie Avances de Investigación, $\mathrm{N}^{\circ}$ 62, Madrid, Fundación Carolina-CeALCI

Gayo, M., B. Teitelboim y M.L. Méndez (2013), "Exclusividad y fragmentación: los perfiles culturales de la clase media en Chile", Universum, vol. 1, $\mathrm{N}^{\circ} 28$, Universidad de Talca.

(2009), "Patrones culturales de uso del tiempo libre en Chile. Una aproximación desde la teoría bourdieuana", Universum, vol. 24, $\mathrm{N}^{\circ}$ 2, Universidad de Talca.

Gerhards, J., S. Hans y M. Mutz (2013), "Social class and cultural consumption: the impact of modernisation in a comparative European perspective", Comparative Sociology, vol. 12, $\mathrm{N}^{\circ} 2$.

Graaf, N.D. y B. Steijn (1996), "The service class in a post-industrial society. Attitudes and behaviour of the social and cultural specialists in the public sector", documento presentado en la vigésimo octava reunión de la Asociación Internacional de Sociología.

Güell, P. y T. Peters (eds.) (2012), La trama social de las prácticas culturales. Sociedad y subjetividad en el consumo cultural de los chilenos, Santiago, Ediciones Universidad Alberto Hurtado.

Güell, P., T. Peters y R. Morales (2012), "Derechos culturales y aseguramiento en el acceso al consumo cultural: aplicación empírica de una canasta básica de consumo cultural para Chile", La trama social de las prácticas culturales. Sociedad y subjetividad en el consumo cultural de los chilenos, P. Güell 
y T. Peters (eds.), Santiago, Ediciones Universidad Alberto Hurtado.

Herring, C. (1989), Splitting the Middle. Political Alienation, Acquiescence, and Activism among America's Middle Layers, Nueva York, Praeger.

Knowledge Wharton (2008), "The New Global Middle Class: Potentially Profitable - But Also Unpredictable" [en línea] http://knowledge.wharton.upenn.edu/article/the-new-globalmiddle-class-potentially-profitable-but-also-unpredictable/.

León, A. y J. Martínez (2004), "La estratificación social chilena hacia fines del siglo xx", serie Políticas Sociales, $\mathrm{N}^{\circ} 52$ (LC/L.1584-P), Santiago, Comisión Económica para América Latina y el Caribe (CEPAL).

López-Calva, L.F. y E. Ortiz-Juárez (2012), "Clases medias y vulnerabilidad a la pobreza en América Latina", Pensamiento Iberoamericano, $\mathrm{N}^{\circ} 10$.

Lora, E. y J. Fajardo (2011), Latin American Middle Classes: The Distance between Perception and Reality, Washington, D.C., Banco Interamericano de Desarrollo.

Lury, C. (2000), Consumer Culture, Cornwall, Polity Press.

Marx, C. y F. Engels (1971), Manifiesto del partido comunista, Pekín, Ediciones en Lenguas Extranjeras.

McAdams, J. (1987), "Testing the theory of the new class", The Sociological Quarterly, vol. 28, $\mathrm{N}^{\circ} 1$, Wiley.

Méndez, M.L. (2010), "Clases medias en Chile: transformaciones, sentido de pertenencia y tensiones entre proyectos de movilidad", Las clases medias en América Latina, R. Franco, M. Hopenhayn y A. León (coords.), Santiago, Ciudad de México, Siglo Xxi/Comisión Económica para América Latina y el Caribe (CEPAL).

(2008), "Middle class identities in a neoliberal age: tensions between contested authenticities", The Sociological Review, vol. $56, \mathrm{~N}^{\circ} 2$, Wiley.

(2007), "Do I See Myself as Others See Me? Middle Class Identities in Chile", tesis, Manchester, Universidad de Manchester

Méndez, M.L. y M. Gayo (2007), "El perfil de un debate: movilidad y meritocracia. Contribución al estudio de las sociedades latinoamericanas", Estratificación y movilidad social en América Latina. Transformaciones estructurales de un cuarto de siglo, R. Franco, A. León y R. Atria (coords.), Santiago, LOM Ediciones/Comisión Económica para América Latina y el Caribe (CEPAL).

Mills, C.W. (1959), White Collar. The American Middle Classes, Nueva York, Oxford University Press.

Nazif, J.I. (2007), "Diferenciación social en patrones de consumo de exposiciones de arte, teatro, danza, recitales en vivo y circo en la sociedad chilena", Revista de Sociología, № 21, Santiago, Universidad de Chile.

Nivón, E. y D. Sánchez (2012), "Convergencias en México y Chile: entornos y estudios de consumo cultural", La trama social de las prácticas culturales. Sociedad y subjetividad en el consumo cultural de los chilenos, P. Güell y T. Peters, Santiago, Ediciones Universidad Alberto Hurtado.
O'Dougherty, M. (2002), Consumption Intensified: The Politics of Middle-Class Daily Life in Brazil, Durham, Duke University Press.

oCDE (Organización para la Cooperación y el Desarrollo Económicos) (2011), Perspectivas económicas de América Latina 2011. En qué medida es clase media América Latina, OECD Publishing.

OEI (Organización de Estados Iberoamericanos para la Educación, la Ciencia y la Cultura) (2014), Encuesta Latinoamericana de hábitos y prácticas culturales 2013, Madrid.

Pakulski, J. y M. Waters (1996), The Death of Class, Thousand Oaks, SAGE.

Paramio, L. y M. Güemes (2013), Cómo son y qué piensan los diferentes estratos de la clase media latinoamericana: caracterización y análisis de percepciones, Madrid, Instituto Universitario Ortega y Gasset.

Perkin, H. (1996), The Third Revolution. Professional Elites in the Modern World, Londres, Routledge.

(1989), The Rise of Professional Society. England since 1880, Londres, Routledge.

Peterson, R.A. y R.M. Kern (1996), "Changing highbrow taste: from snob to omnivore", American Sociological Review, vol. 61, $\mathrm{N}^{\circ}$ 5, American Sociological Association.

Pinto, L. (2013), "Du bon usage de La Distinction", Trente ans après La Distinction de Pierre Bourdieu, Ph. Coulangeon y J. Duval, París, La Découverte.

PNUD (Programa de las Naciones Unidas para el Desarrollo) (2002), Desarrollo humano en Chile. Nosotros los chilenos: un desafio cultural 2002, Santiago.

Rasse, A., R. Salcedo y J. Pardo (2009), “Transformaciones económicas y socioculturales: ¿cómo segmentar a los chilenos hoy?", El arte de clasificar a los chilenos. Enfoques sobre los modelos de estratificación en Chile, A. Joignant y P. Güell (coords.), Santiago, Ediciones Universidad Diego Portales.

Roberts, K. (2004), "Leisure inequalities, class divisions and social exclusion in present-day Britain", Cultural Trends, vol. 13, $\mathrm{N}^{\circ}$ 2, Taylor \& Francis.

Savage, M. y otros (1992), Property, Bureaucracy and Culture. Middle-Class Formation in Contemporary Britain, Londres, Routledge.

Skogen, K. (1996), "Young environmentalists: post-modern identities or middle-class culture?", The Sociological Review, vol. 44 $\mathrm{N}^{\circ}$ 3, Wiley,

Torche, F. (2007), "Social status and cultural consumption: the case of reading in Chile", Poetics, vol. 35, $\mathrm{N}^{\circ}$ 2-3, Amsterdam, Elsevier.

Torche, F. y L.F. López-Calva (2013), "Stability and vulnerability of the Latin American middleclass", Oxford Development Studies, vol. 41, $\mathrm{N}^{\circ}$ 4, Taylor \& Francis.

Wahrman, D. (1995), Imagining the Middle Class. The Political Representation of Class in Britain, c.1780-1840, Cambridge, Cambridge University Press.

Wright, E.O. (1994), Clases, Madrid, Siglo XXI. (1983), Clase, crisis y Estado, Madrid, Siglo XXI. 